\title{
Lanthanide-based time-resolved luminescence immunoassays
}

\author{
A. K. Hagan • T. Zuchner
}

Received: 11 February 2011 /Revised: 12 April 2011 / Accepted: 19 April 2011 /Published online: 11 May 2011

(C) The Author(s) 2011. This article is published with open access at Springerlink.com

\begin{abstract}
The sensitive and specific detection of analytes such as proteins in biological samples is critical for a variety of applications, for example disease diagnosis. In immunoassays a signal in response to the concentration of analyte present is generated by use of antibodies labeled with radioisotopes, luminophores, or enzymes. All immunoassays suffer to some extent from the problem of the background signal observed in the absence of analyte, which limits the sensitivity and dynamic range that can be achieved. This is especially the case for homogeneous immunoassays and surface measurements on tissue sections and membranes, which typically have a high background because of sample autofluorescence. One way of minimizing background in immunoassays involves the use of lanthanide chelate labels. Luminescent lanthanide complexes have exceedingly long-lived luminescence in comparison with conventional fluorophores, enabling the shortlived background interferences to be removed via timegated acquisition and delivering greater assay sensitivity and a broader dynamic range. This review highlights the potential of using lanthanide luminescence to design sensitive and specific immunoassays. Techniques for labeling biomolecules with lanthanide chelate tags are discussed, with aspects of chelate design. Microtitre platebased heterogeneous and homogeneous assays are reviewed and compared in terms of sensitivity, dynamic range, and convenience. The great potential of surface-based timeresolved imaging techniques for biomolecules on gels,
\end{abstract}

A. K. Hagan · T. Zuchner $(\bowtie)$

Institute of Bioanalytical Chemistry, Center of Biotechnology

and Biomedicine, Faculty of Chemistry and Mineralogy,

Leipzig University,

Deutscher Platz 5,

04103 Leipzig, Germany

e-mail: zuechner@rz.uni-leipzig.de membranes, and tissue sections using lanthanide tracers in proteomics applications is also emphasized.

Keywords Bioassays · Fluorescence/luminescence · Rare earth elements · Genomics/proteomics · Bioanalytical methods

\section{Introduction}

The detection of particular analytes is the cornerstone of analytical chemistry and is crucial for a plethora of different applications, ranging from protein detection to drug screening and mapping of biochemical pathways. Two vital features of the ideal assay are specificity and sensitivitythat is, the analyte of interest should be:

1. unambiguously detected even when potentially interfering substances are present in excess, and

2. detectable at the low concentrations typically required for most applications.

In protein detection, these problems are especially challenging to solve. First, biological samples are highly complex and contain a huge number of different proteins, making the detection of one specific protein a special challenge. Second, some proteins are expressed in extremely low copy numbers, especially in the early stages of disease. Third, biological samples containing proteins have high background fluorescence, making it difficult to obtain good sensitivity using simple fluorophores. Last, but not least, the difference between high-abundance and low-abundance proteins in one biological sample can span an estimated twelve orders of magnitude [1]. This requires detection methods with broad linear ranges. In general, time-resolved luminescence is an ideal tool to meet all of these challenges. As will be 
discussed later, time-resolved luminescence not only provides excellent detection limits but also broadens the linear range of existing protein detection assays, because of the low background signals associated with this method.

In immunoassays the specificity is provided by antibodies raised against the analyte of interest, whereas sensitivity relies on some form of coupling of analyte-antibody binding with a detectable and quantifiable signal. In the simplest format this involves direct labeling of the antibody with a small molecule tag, for example a radioisotope or luminophore, which can then be detected using appropriate analytical devices. A variety of assay formats are also possible, including well plate-based assays in which the antigen of interest is either immobilized on the well plate surface or present in solution, membrane-based assays in which proteins are separated by gel electrophoresis and subsequently transferred to a membrane for detection, and direct staining of tissue sections for examination under a fluorescence microscope.

The objective of this critical review is to highlight the potential of lanthanide-based time-resolved luminescence for developing ultrasensitive immunoassays. The basic principles behind immunoassays (in the section "Immunoassays") and time-resolved luminescence measurements (in the section "Lanthanide luminescence") will be discussed, followed by a thorough overview of the main techniques for labeling biomolecules with lanthanides via various chelating groups (in the section "Chelator design and lanthanide labeling of biomolecules"). The remainder of the review will focus on reported immunoassays using lanthanide-based luminescence detection and will be divided into two main sections: the well-established field of microtitre plate-based assays, encompassing heterogeneous and homogeneous assays (the section "Microtitre plate-based assays"), and the nascent but promising field of imaging-based methods such as membrane imaging and time-resolved luminescence microscopy (in the section "Surface-based assays").

\section{Immunoassays}

The term "immunoassay" refers to the use of antibodies raised against an analyte of interest (antigen) as a means of detecting the presence of that analyte. Because neither antibodies nor their binding to their antigens have high intrinsic detectability, the use of antibodies as detection reagents requires some form of labeling of the antibody with an easily detectable reporter molecule. The conceptually simplest reporters consist of antibodies directly labeled with either an enzyme or small molecule light absorbers, fluorophores, or radioisotopes, although indirect labeling via a species-specific secondary antibody or the biotinstreptavidin interaction results in greater versatility and some signal amplification.
The first immunoassays used antibodies labeled with radioisotopes that could subsequently be detected by use of scintillation counters. Although highly sensitive, the problems associated with radioisotopes (limited stability, safe disposal, etc.) have led to the development of alternative detection strategies, of which the most widespread is currently the use of antibody-enzyme conjugates, in which enzymatic turnover converts an uncolored substrate to a colored product which can be quantified colorimetrically. These assays have the advantage of amplification - that is, conversion of a single antibody-enzyme immunocomplex into multiple detectable molecules. However, the linear range of colorimetric detection is limited and the colordevelopment process necessitates additional time, steps, and reagents. Antibody-fluorophore conjugates have the potential to eliminate some of these problems, because the fluorescence of the immunocomplex can be measured directly on the surface or in solution. However, they suffer from the disadvantage that no amplification occurs, requiring very bright luminescence output from each luminophore or high degrees of labeling. The latter can be difficult to achieve without compromising antibody activity or solubility. One way of achieving high sensitivity in luminescence-based assays entails the use of time-resolved luminescence measurements to minimize the sample background. The advantages of using time-resolved luminescence for immunodetection will be the focus of this review.

Four basic assay configurations are possible, depending on whether the immunoreaction occurs on a solid phase (heterogeneous) or in solution (homogeneous) and whether the antibody-analyte binding is monitored directly (noncompetitive) or via displacement of a labeled antigen (competitive). Typical solid phases for a heterogeneous immunoassay include microtitre plates, membranes and tissue slices, to which the antigen is either bound directly or on which it is captured by use of an appropriate antibody (Fig. 1). The presence of this antigen can then be determined after binding an appropriately labeled antibody (or primary-secondary antibody combination) and measuring, e.g., the luminescence signal. A typical homogeneous non-competitive assay utilizes two differently labeled antibodies which interact with one another in some way (e.g. by luminescence resonance energy transfer, LRET) in the presence of the target antigen (Fig. 1), whereas a homogeneous competitive assay could involve a system whereby the target antigen displaces a labeled antigen analogue from a labeled antibody and thereby reduces the LRET intensity.

\section{Lanthanide luminescence}

Luminescence is the process in which a species absorbs light at one wavelength and subsequently emits it at a 

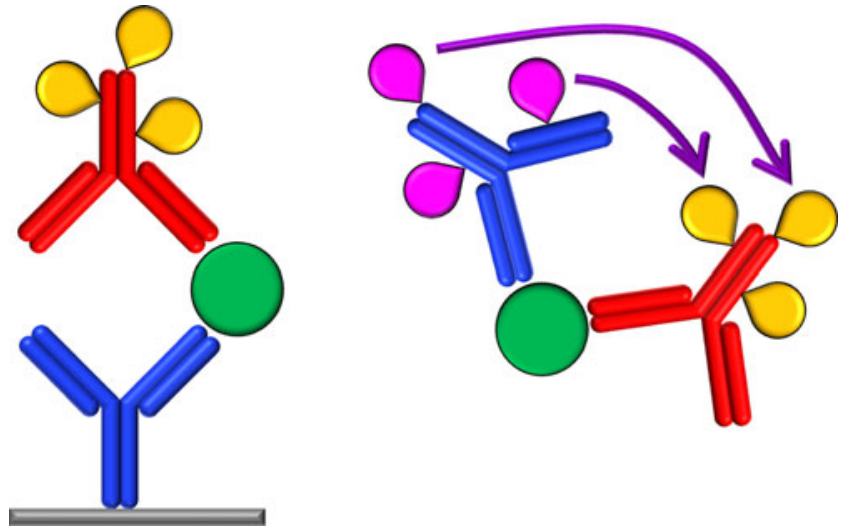

Fig. 1 Two representative immunoassay formats - a heterogeneous sandwich immunoassay (left) and a homogeneous immunoassay based on LRET between two luminescent labels (indicated by purple arrow; right). Antibodies labeled with tags (teardrop) are used for detection of antigens (circles)

second, generally higher, wavelength. The energy absorption excites the molecule from its ground state singlet electronic level $\left(\mathrm{S}_{0}\right)$ to a higher level $\left(\mathrm{S}_{1}, \mathrm{~S}_{2}\right.$, etc. $)$ as shown in Fig. 2. Molecules excited to upper singlet energy levels $\left(\mathrm{S}_{2}\right.$ etc.) rapidly $\left(\sim 10^{-10} \mathrm{~s}\right)$ return to the lowest excited state $\mathrm{S}_{1}$ without light emission, from which a number of radiative (fluorescence) and non-radiative transitions can occur to return the molecule to any of the rotational and/or vibrational levels of the ground electronic state $\mathrm{S}_{0}$. The transition from $S_{1}$ to the triplet state $T_{1}$ is referred to as intersystem crossing and can be followed by relaxation to $\mathrm{S}_{0}$ either radiatively (phosphorescence) or non-radiatively. In some circumstances $T_{1}$ can transfer the excitation energy to other species, for example chelated lanthanide ions $\left(\mathrm{Eu}^{3+}\right.$, $\left.\mathrm{Tb}^{3+}, \mathrm{Dy}^{3+}, \mathrm{Sm}^{3+}\right)$, from which ion luminescence can occur [2]. This ligand-ion energy transfer is known as the antenna effect, and enables the observation of relatively intense luminescence signals from lanthanide ions, which absorb energy only poorly in the absence of ligand. $\mathrm{Eu}^{3+}$, the lanthanide ion that is most commonly used in bioanalytical applications, can accept energy from the ligand triplet state into any of several lower energy ${ }^{5} \mathrm{D}_{j}$ levels, with emission then occurring via transition from ${ }^{5} \mathrm{D}_{0}$ to any of the groundstate levels ${ }^{7} \mathrm{~F}_{j}$ to give signals at several characteristic wavelengths (Fig. 2) [3]. The efficiency of this energy transfer and the intensity of the subsequent emission depend on both the ligand and the ion in question, and in particular on the energy gap between the ligand triplet level and the ion accepting level and the ability of the ligand to shield the ion from quenching inner sphere water molecules, as discussed later from the perspective of ligand design (in the section "Chelator design and lanthanide labeling of biomolecules").

The key feature of lanthanide luminescence that renders it interesting bioanalytically is that lanthanide ions have exceedingly long-lived luminescence ( $\mu \mathrm{s}$ to $\mathrm{ms}$ range), because the corresponding transitions are Laporteforbidden. In comparison, luminescence from conventional fluorescent dyes and sample interferences occurs on a nanosecond scale. This unique feature means that lanthanide luminescence can be selectively measured even in the presence of other luminescent substances by time-gating the signal - that is, by only starting the acquisition cycle after the more rapid background fluorescence has decayed (Fig. 3). The benefit of this in terms of assay development is that the sensitivity of an assay depends strongly on the signal-to-background ratio that can be achieved. A high background signal for blank control samples containing no analyte worsens the limits of detection and quantification for real samples and limits the linear quantification range of an assay. Background in luminescence measurements arises from various sources, including the sample vessel or membrane, but the most important source in biological assays is generally the sample matrix. This is particularly the case for homogeneous assays in which interfering substances are not removed before measurement. A typical biological sample, for example serum or a tissue sample,
Fig. 2 Luminescence emission processes of a general europium chelate. An antenna ligand absorbs the excitation energy and transfers it to the $\mathrm{T}_{1}$ level via intersystem crossing, from which it is transferred to the chelated $\mathrm{Eu}^{3+}$ and emitted via several transitions

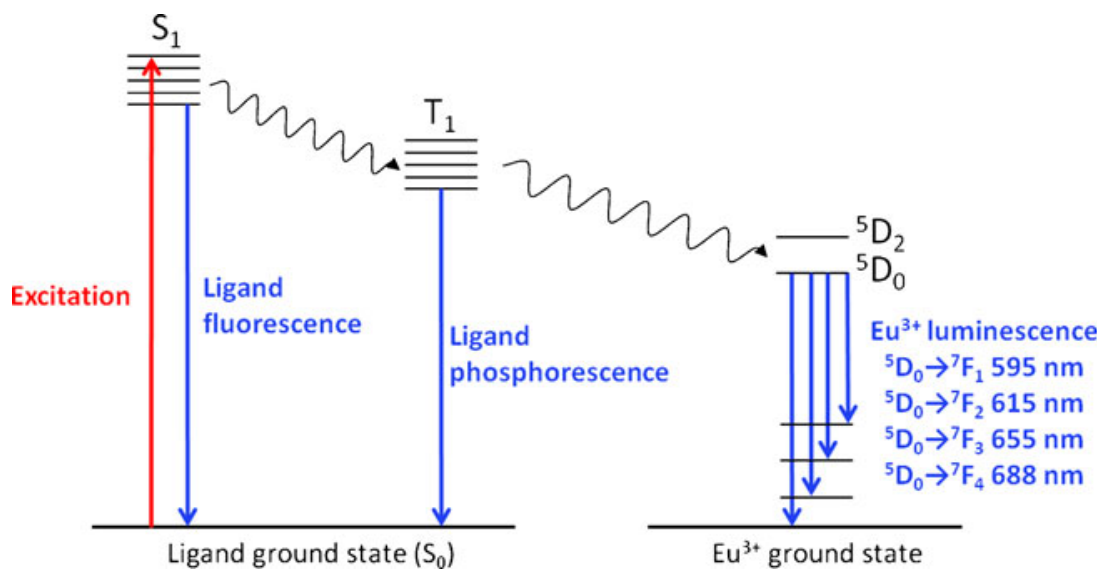




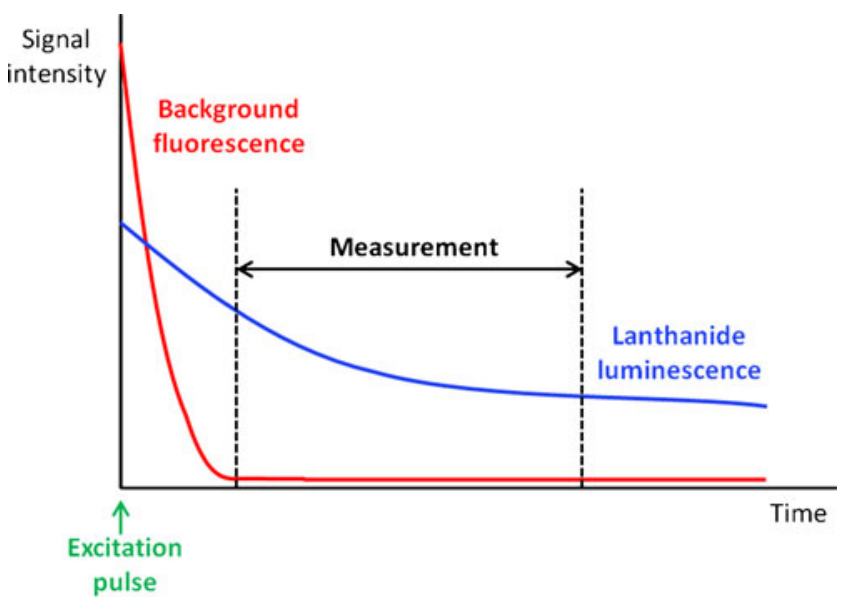

Fig. 3 General principle of time-resolved luminescence measurements

contains a multitude of luminescent substances, for example proteins and $\mathrm{NAD}^{+} / \mathrm{H}[4,5]$, and the detection limits of various fluorophore-protein conjugates in serum have been reported to be 50 to 1000 -fold higher than in buffer [6]. However, the vast majority of these interferences have standard short-lived fluorescence and can be filtered out by time-gated detection. This also has the consequence of broadening the linear range that can be achieved. Scattering of the excitation light by the instrument optics, sample vessel, and matrix is an additional problem with conventional fluorophores, because of their generally small Stokes shifts $(<50 \mathrm{~nm})$; this is circumvented by using lanthanide chelates $(>150 \mathrm{~nm})$. A final problem with conventional fluorophores is self-quenching because of substantial overlap between their excitation and emission spectra, meaning that multiply-labeled biomolecules fluoresce much less than might be expected from the degree of labeling. With their large Stokes shifts, narrow emission bands, and no overlap between excitation and emission spectra, lanthanide chelates are not susceptible to this problem and so are ideal candidates for highly sensitive proteindetection assays.

In the field of lanthanide-based time-resolved luminescence (TRL) immunoassays, the most common solid phases are microtitre plates. This has the practical advantage in terms of instrumentation that TRL capability can be incorporated into luminescence plate readers that are also capable of measuring other luminescence phenomena, for example fluorescence polarization or chemiluminescence. This provides a convenient and cost-effective measurement solution for the end user. A number of different plate readers with TRL measurement modes exist today. They vary in respect of the type of excitation sources, filters and/or monochromators, time-gating, and focus setups used. Excitation sources can be either flash lamps, lasers or UV-LEDs, although combinations of excitation sources (flash lamp plus UV-LED) do exist in some instruments in order to combine sensitivity with high flexibility (e.g. SpectraMax Paradigm, Molecular Devices). In particular, the strong $337 \mathrm{~nm}$ emission of a nitrogen laser is suitable for most europium chelates. Depending on the excitation source, instruments are either filter-based or monochromator-based. Whereas filter-based devices are generally regarded as more sensitive, they are not as flexible as monochromator-based devices (e.g. the Infinite m1000 system from Tecan). The time-delayed detection of the fluorescence signal is normally achieved by oscillatorcontrolled electronic devices. The strategy here is to ignore photon counts which arrive at the detector before a specific timepoint. A general challenge in TRL readers is the need for photomultiplier tubes with exceptionally low dark count rates. This is because the photon output of lanthanide-based TRL dyes is generally low compared with that of conventional fluorophores. With the recent development of various lanthanide based immunoassays as described below, more and more TRL-capable plate readers are becoming commercially available and more users are equipped to perform the assays described below.

\section{Chelator design and lanthanide labeling of biomolecules}

\section{Introduction}

The use of lanthanide ions in immunoassays requires their incorporation into the immunocomplex. This is achieved via the use of chelating agents, either by direct labeling of antibodies or by indirect labeling of secondary detection reagents. One secondary detection reagent is streptavidin, which can then be used with readily available biotinylated antibodies. A lanthanide chelator for use in immunoassays therefore consists of at least two parts - a chelating group for binding the lanthanide ion and some functionality for attachment to biomolecules. When the lanthanide-labeled biomolecule must be intrinsically luminescent, an additional sensitizing antenna moiety is required (as described in the section "Lanthanide luminescence"). The need to integrate the optimum requirements of each of these three components into a single molecule - that is, to create a stable complex which combines efficient light absorption, energy transfer, and emission, and which can be conveniently attached to biomolecules without interfering with their activity-is a major challenge in this field of research and the key points will be introduced in the section "Ligand requirements". Numerous examples of specific lanthanide chelates from several structural classes will then be introduced in the sections "Non-luminescent chelates", "PAC-based luminescent chelates", " $\beta$-Diketone-based luminescent chelates", and "Other luminescent chelates"). The focus here will be 
on chelating structures that have been applied or have the potential to be applied to immunoassays rather than those designed for, e.g., electroluminescent materials applications, which have been excellently reviewed elsewhere [7].

Ligand requirements

\section{Chelating groups}

To incorporate lanthanide ions into luminescent complexes, chelating groups are required. Trivalent lanthanide cations $\mathrm{Ln}^{3+}$ are hard Lewis acids with a coordination number of 8-9 [8], requiring hard donors, for example carboxylates, $\beta$-diketonates and phosphonates, which contain nitrogen and oxygen donor atoms. Although lanthanide ions can be complexed by monodentate or bidentate ligands (e.g. 2thenoyltrifluoroacetone, salicylic acid) and such complexes find application in some assays (described in the section "Heterogeneous assays"), most practical systems rely on the ability to carry the lanthanide ion as a "tag" on the biomolecule through the various assay steps. This implies that the complex has sufficient stability under the various assay conditions, and generally necessitates the use of multidentate chelators.

One major class of such chelators are the polyaminocarboxylates (PACs), for example the linear ethylenediaminetetraacetic acid (EDTA) and diethylenetriaminepentaacetic acid (DTPA) and their cyclic counterparts 1,4,7,10-tetraazacyclododecane-1,4,7,10-tetraacetic acid (DOTA) and 1,4,8,11- tetraazacyclotetradecane-1,4,8-triacetic acid (DO3A) (Fig. 4). These are the archetypal metal-sequestering agents that are used in a range of industrial applications and are readily available, inexpensive, and form soluble and highly stable complexes with lanthanides under basic conditions. The high stability of the lanthanide-PAC complexes stems from the ability of the multiple amine and carboxylate coordinating centers to wrap around the metal ion, and increases in the order EDTA $<$ DTPA $<$ DOTA with the denticity and from $\mathrm{La}$ to $\mathrm{Lu}$ across the lanthanide series with the decreasing ionic radius, with typical $\log K$ values for the lanthanide complexes being $10^{15}-10^{20}, 10^{19}-10^{23}$, and $10^{23}-10^{26}$ for EDTA, DTPA, and DOTA complexes, respectively [9]. An additional advantage for the higherdenticity PACs is that saturation of the lanthanide coordination sphere by the ligand excludes water and thereby minimizes aqueous quenching. These ligands also contain several possible derivatization points for installation of antenna moieties and reactive groups (see below).

A second group of lanthanide chelators that have found application in immunoassays are $\beta$-diketonates derived from the corresponding $\beta$-diketones under basic conditions (Fig. 4). Simple $\beta$-diketonates have two oxygen atoms available for coordination and tend to form 3:1 complexes with lanthanides, with the remaining vacant sites in the lanthanide coordination sphere being occupied by water molecules. Many of these ligands include aromatic antenna groups (see below) and their lanthanide complexes are intrinsically luminescent. Although a large number of lanthanide $\beta$ -
Fig. 4 Selected chelating, antenna, and protein-reactive groups for labeling of biomolecules with lanthanides<smiles>O=C(O)CN(CCN(CCOC(=O)CN(CCN(CC(=O)O)CC(=O)O)CC(=O)O)CCN(CC(=O)O)CC(=O)O)CC(=O)O</smiles><smiles>[R2]C(=O)C=C([R2])O</smiles>

EDTA

DTPA<smiles>c1ccc(-c2ccccn2)nc1</smiles>

2,2'-bipyridine
DOTA

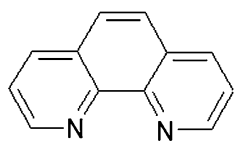

1,10-phenanthroline<smiles>c1ccncc1</smiles>

pyridine
Protein-reactive groups

$\mathrm{R}-\mathrm{SO}_{2} \mathrm{Cl}$

$\mathrm{R}-\mathrm{SCN}$

sulfonyl chloride

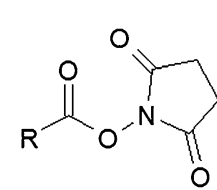

NHS ester<smiles>[R]C(=O)CI</smiles>

iodoacetamide<smiles>[R]N1C(=O)C=CC1=O</smiles>

maleimide 
diketonates have been synthesized [10], only a minority of these are useful as lanthanide tags for biomolecules, because each $\beta$-diketone is only bidentate and the complexes therefore have limited stability. In an attempt to overcome this limitation, several tetradentate ligands have been created which combine two $\beta$-diketones in one molecule, and these species are sufficiently stable for use in immunoassays (discussed in the section "PAC-based luminescent chelates"). It is also worth noting here that, despite their limited stability, the bidentate ligands are a crucial part of the most successful lanthanide-based immunoassay reported to date - the DELFIA system-where they serve as solution ligands after dissociation of lanthanide tags from a non-fluorescent chelate-containing immunocomplex (discussed in the section "Heterogeneous assays"). In particular, $\beta$-naphthoyltrifluoroacetone and 2-thenoyltrifluoroacetone form intensely luminescent complexes with $\mathrm{Eu}^{3+}$ and $\mathrm{Tb}^{3+}$, although a minor disadvantage of these ligands compared with PACs is that it is usually necessary to use an auxiliary ligand such as trioctylphosphine oxide (TOPO) in order to saturate the remaining sites in the lanthanide coordination sphere and exclude water [11, 12].

Several additional lanthanide chelates beyond these two main classes have also been reported; these bind to lanthanides via multiple carboxylates and one or more aza-aromatic groups (e.g. pyridine, 2,2'-bipyridine, 1,10phenanthroline) which function both as chelator and antenna. This has been termed the chromophoric chelate strategy [13], and includes the tris-bipyridyl cryptands [14] and BCPDA $\left(\varepsilon \approx 15,200 \mathrm{~mol}^{-1} \mathrm{~L} \mathrm{~cm}^{-1}\right.$ (325 nm)) [15] (see below). By comparison, the pendant chromophore strategy involves the grafting of one or more chromophores on to one of the aforementioned multidentate chelators, for example DTPA or DO3A. Two examples of ligands developed using this strategy are DTPA-cs124 $(\varepsilon \approx$ $\left.8000 \mathrm{~mol}^{-1} \mathrm{~L} \mathrm{~cm}^{-1}(337 \mathrm{~nm})\right)$ [16] and [EuL]H [17]. Although both strategies can lead to effective chelators, the chromophoric chelate approach is considered preferable because it places the antenna function in close proximity to the metal and thereby facilitates rapid energy transfer [18]. Chromophoric chelates do however typically require augmenting agents, for example $\mathrm{F}^{-}$, to exclude water from the first coordination sphere and optimize the luminescence [19]. Furthermore, for applications requiring the highest chelate stability the pendant chromophore strategy using multidentate PACs and an appropriate sensitizer is probably optimum (as discussed in the section "PAC-based luminescent chelates"). In general, an optimum chelator for all kinds of different systems does not seem to exist. All of the chelator structures introduced here have been successfully used to construct luminescent chelates for use in sensitive assays, as will be discussed later (in the section "Microtitre plate-based assays").

\section{Antennas}

After incorporation of a suitable lanthanide ion into a chelating complex, antenna regions are required to efficiently transfer excitation energy to the lanthanide ion. Such antenna groups consist of an highly $\pi$-conjugated aromatic or heteroaromatic system which efficiently absorbs light and transfers it to the lanthanide ion, increasing the molar extinction coefficient from ca 1$10 \mathrm{~mol}^{-1} \mathrm{~L} \mathrm{~cm}^{-1}$ for the uncomplexed trivalent cation to ca $10^{4}-10^{5} \mathrm{~mol}^{-1} \mathrm{~L} \mathrm{~cm}^{-1}$ for a typical lanthanide-antenna complex $[18,20]$. Typical antenna ligands include naphthalene, 1,10-phenanthroline, and 2,2'-bipyridine (Fig. 4). A systematic study of the complexes of 41 ligands with $\mathrm{Eu}^{3+}$ and $\mathrm{Tb}^{3+}$ revealed that the energy of the ligand triplet excited state is crucial in determining the sensitization efficiency of an antenna and should be at least $1,850 \mathrm{~cm}^{-1}$ higher than the lowest excited state of the lanthanide ion [21]. A smaller energy gap leads to a reduced quantum yield because of back energy transfer and quenching of the antenna triplet level, whereas a higher energy gap is acceptable because the energy transferred from the antenna transitions through the non-radiative excited states until it reaches the emittive level [18]. This energy gap requirement means that the choice of antenna depends somewhat on the lanthanide ion being used, and the optimum antenna for one lanthanide ion is seldom the optimum choice for another. In addition it is highly desirable that the excitation maximum of the antenna is above ca $350 \mathrm{~nm}$, which enables the use of less expensive glass rather than quartz optics and avoids the excitation of other chromophores (e.g. Trp, NADH) in biological media. This is still a challenge in the development of new lanthanide-based dyes.

\section{Reactive groups for coupling to biomolecules}

There are essentially only two generally applicable chemical strategies for selective and efficient covalent attachment of luminophores or other small molecules to proteins - via either Lys amino groups (using e.g. active esters, isothiocyanates) or Cys thiols (using e.g. maleimides, iodoacetamide) (Fig. 4). These activated derivatives are ideally relatively stable under anhydrous conditions and can be conveniently coupled to proteins at neutral to slightly basic $\mathrm{pH}$. Unlike conventional fluorophores, lanthanide chelates are seldom susceptible to self-quenching, and so it is generally desirable to incorporate as many lanthanide labels as possible into the final immunocomplex as can be achieved without affecting the immunological binding or physical properties (e.g. solubility). The maximum labeling degree that can be achieved depends on the protein, chelate, and reaction conditions in question, but 4-15 labels for a $150 \mathrm{kDa}$ antibody using a simple PAC derivative is typical [22]. 
An alternative strategy to maximize the number of labels per antibody involves the use of labeled streptavidin as a universal detection system, because its binding affinity for biotin seems to tolerate higher labeling degrees, in addition to enabling a multivalency effect via multiply biotinylated antibodies [12, 23-26]. In a particularly extreme case, a macromolecular conjugate consisting of streptavidin, three units of thyroglobulin, and 480 lanthanide chelates has been reported [27, 28].

A novel recent alternative to labeling biomolecules with luminescent chelates that has recently emerged is the surface coating of a submicrometer luminescent bead with the biomolecule. Polystyrene beads (107 nm) containing $>30,000$ europium(III)-2-thenoyltrifluoroacetone complexes per particle are commercially available and have a carboxylic acid surface which can be used for covalent attachment of streptavidin or antibodies [29, 30]. The hydrophobic interior of such beads minimizes aqueous quenching and high luminescence intensities are obtained.

Although some of the labeling strategies lead to higher numbers of lanthanide dyes per protein than others, it is clear that coupling of lanthanide dyes to proteins is now a very well established technique and not a major challenge in terms of lanthanide dye compatibility in the field of immunoassays.

\section{Non-luminescent chelates}

The concept of using lanthanide chelates as labels in immunoassays was suggested thirty years ago by Wieder [31] and then by Soini and Hemmilä [6], and finally realized in 1983 with the advent of the DELFIA system [32]. This relies on a non-luminescent lanthanide tag which is liberated before measurement by addition of an enhancement solution. As such, simple reactive EDTA or DTPA-derived chelators which lack an antenna function can be utilized, for example cyclic DTPA anhydride (cDTPAA), $p$-SCN-Bn-DTTA, and $p$-SCN-Bn-DTPA (Fig. 5). A compromise is required here between the need for chelate stability during storage and washing steps and rapid release of the lanthanide before measurement; one study revealed $p$-SCN-Bn-DTTA to be the optimum choice of those tested [33]. In this case, labeling degrees of 15-25 $\mathrm{Eu}^{3+}$ chelates per $\mathrm{IgG}$ were achieved and dissolution was achieved within 1 min of addition of the enhancement solution. Although $c$-DTPAA is a readily available and inexpensive alternative, it is poorly soluble and the reaction is difficult to control and may result in crosslinking of the biomolecule. A method for utilizing DOTA derivatives in the DELFIA system has also recently been reported; this uses a treatment step with $2.0 \mathrm{~mol} \mathrm{~L}-1$ $\mathrm{HCl}$ to release lanthanides from these more stable chelates before measurement [34]. A major advantage of the DELFIA system is the relative ease with which multiplexing can be achieved, because the same simple chelates can be used with any of the luminescent lanthanide ions, assuming enhancement solutions are available for all lanthanides present (typically $\mathrm{Eu}^{3+}, \mathrm{Tb}^{3+}, \mathrm{Dy}^{3+}, \mathrm{Sm}^{3+}$ ).

PAC-based luminescent chelates

A convenient means of preparing luminescent lanthanide chelates is to attach an antenna moiety to one of the carboxylates of DTPA, because the resulting product still has sufficiently high denticity to form stable chelates. Reaction of $c$-DTPAA (Fig. 5) with the commercially-available sensitizer carbostyril $124\left(\varepsilon=12,000 \mathrm{~mol}^{-1} \mathrm{~L} \mathrm{~cm}^{-1}(327 \mathrm{~nm})\right)$ afforded DTPA-cs124 (Fig. 6), which forms luminescent complexes with both $\mathrm{Eu}^{3+}$ and $\mathrm{Tb}^{3+}[35]$. The remaining anhydride of $c$ DTPAA can also be reacted with diamines and further converted to an isothiocyanate or maleimide derivative to enable facile coupling to proteins or DNA [36, 37]. Likewise, the recent synthesis of the ligand $[\mathrm{EuL}] \mathrm{H}$ (Fig. 6) relied on attachment of a 1,10-phenanthroline-based sensitizer to $c$-DTPAA and subsequent reaction of an additional carboxylate with a maleimide derivative [17]. Coupling of the maleimide to proteins and incubation with $\mathrm{Eu}^{3+}$ enabled the detection of 100 amol lysozyme on PVDF membranes and 1.5 fmol BSA in SDS-PAGE gels, and a linear range spanning five orders of magnitude was
Fig. 5 Non-luminescent lanthanide chelators for use in DELFIA assays

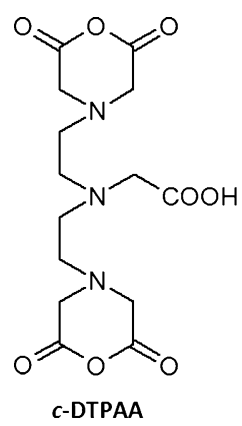<smiles>N#S(=O)c1ccc(CN(CCN(CCN(CC(=O)O)CC(=O)O)CC(=O)O)CCN(CC(=O)O)CC(=O)O)cc1</smiles>

p-SCN-Bn-DTTA
p-SCN-Bn-DTPA 

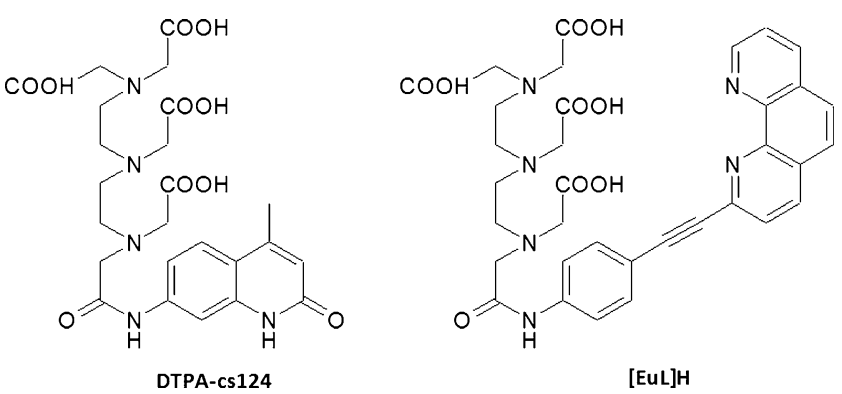

Fig. 6 Luminescent PAC-based lanthanide chelators

demonstrated. The $[\mathrm{EuL}] \mathrm{H}$ excitation spectrum covers an unusually wide range, making it compatible with a wide range of excitation sources from 310-365 nm.

\section{$\beta$-Diketone-based luminescent chelates}

Although simple $\beta$-diketones do not form sufficiently stable complexes to be used as labeling reagents, incorporation of two $\beta$-diketones into one molecule gives a tetradentate ligand which can be successfully used in immunoassays. The $\beta$-diketone units are held in place by a rigid or flexible spacer and also contain an aromatic or heteroaromatic antenna function; several such ligands have been developed (Fig. 7). The first example was BCDOT, which contains two biphenyl antennas for efficient light absorption $\left(\varepsilon=36,500 \mathrm{~mol}^{-1} \mathrm{~L} \mathrm{~cm}^{-1}(330 \mathrm{~nm})\right)$ and two sulfonyl chloride moieties for coupling to proteins [38]. For

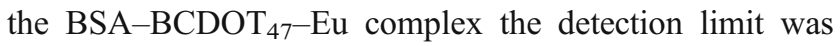

83 fmol $\mathrm{L}^{-1}$ in TOPO-SDS- $\mathrm{NaHCO}_{3}$ solution and the method was shown to be linear over at least four orders of magnitude. The same authors subsequently developed the ligand BCOT, which was found to have similar properties for the free complex $\left(\varepsilon=32,000 \mathrm{~mol}^{-1} \mathrm{~L} \mathrm{~cm}^{-1}(330 \mathrm{~nm})\right)$ and the $\mathrm{BSA}-\mathrm{BCOT}_{40}-\mathrm{Eu}$ conjugate (detection limit 95 fmol L ${ }^{-1}$ ) [39]. In both cases the luminescence intensity was enhanced by the synergistic TOPO ligand, partially quenched by phosphate, and resistant to dissolved oxygen. The thiophene-containing BCTOT has been used similarly [40]. All three ligands suffer from the disadvantage that the two sulfonyl chloride groups can potentially cause crosslinking of the proteins being labeled, although this can be avoided by prior blocking of one group by use of simple amines [38].

A second series of ligands containing more rigid terphenyl structures between the two $\beta$-diketones has also been developed (Fig. 7). A detection limit of $6.5 \mathrm{fmol} \mathrm{L}^{-1}$ was reported for $\mathrm{BSA}-\mathrm{BHHCT}_{35}-\mathrm{Eu}$ [41]. Subsequent work revealed that the luminescence intensity could be increased eightfold by exchanging the $\mathrm{C}_{3} \mathrm{~F}_{7}$ groups in BHHCT for $\mathrm{CF}_{3}$ groups in BTBCT [42]. Potential drawbacks of these ligands include their poor aqueous solubility and the high reactivity of the sulfonyl chloride group, which may lead to poor reagent stability during storage or result in excessive labeling degrees and loss of bioactivity of the labeled molecules. In an attempt to develop a more mild reagent, the NHS ester derivative BHHST has also been prepared and found to have improved solubility and<smiles>[R]C(=O)CC(=O)c1ccc(-c2ccc([X])cc2-c2ccc(C(=O)CC([R])=O)cc2)cc1</smiles><smiles>[R1]C(=O)CC(=O)C(F)(F)C(F)(F)C(F)(F)C(F)(F)C(=O)CC([R1])=O</smiles>

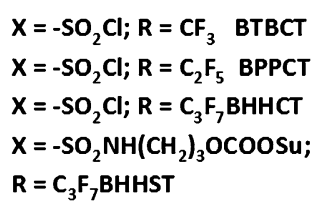

$\mathbf{R 1}=$

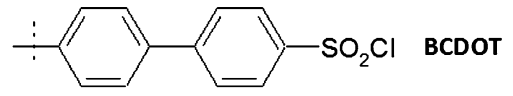

$\mathbf{R} \mathbf{1}=$

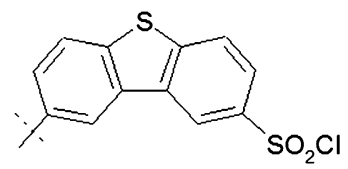

$\mathbf{R} \mathbf{1}=$

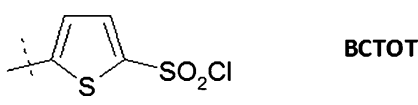

Fig. $7 \beta$-diketonate-based lanthanide chelating agents. Abbreviations: BTBCT, 4,4'-bis(1", 1", 1"-trifluoro-2",4"-butanedion-4"-yl)-chloro-

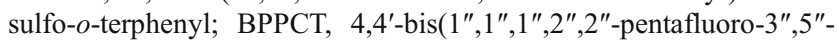
pentanedion-5"-yl)-chlorosulfo-o-terphenyl; BHHCT, 4,4'-bis (1",1", 1",2",2",3",3"-heptafluoro-4",6"-hexanedion-6"-yl)-chlorosulfo-o-terphenyl; BHHST, 4,4'-bis(1", 1", 1",2",2",3",3"-heptafluoro-

4",6"-hexanedion-6"-yl)-sulfonylaminopropyl ester- $N$-succinimide ester-o-terphenyl; BCDOT, 1,10-bis(4"-chlorosulfo-1,1"-diphenyl-4'yl)-4,4,5,5,6,6,7,7,-octafluorodecane-1,3,8,10-tetraone; BCOT, 1,10bis(8'-chlorosulfodibenzothiophene-2'-yl)-4,4,5,5,6,6,7,7,-octafluorodecane-1,3,8,10-tetraone; BCTOT, 1,10-bis(5'-chlorosulfothiophene2'-yl)-4,4,5,5,6,6,7,7,-octafluorodecane-1,3,8,10-tetraone 
reagent and immunoconjugate stability compared with BHHCT [43]. Some of these $\beta$-diketone ligands have also been successfully used to generate sensitive immunoassays, which will be the focus of the section "Surface measurement of directly luminescent chelates".

Other luminescent chelates

BCPDA is a sulfonyl chloride-activated bathophenanthroline derivative (Fig. 8) and has been commercialized as part of the CyberFluor FiaGen product line [15]. The BCPDAEu complex has a moderate extinction coefficient at $325 \mathrm{~nm}$ $\left(\varepsilon \approx 15,200 \mathrm{~mol}^{-1} \mathrm{~L} \mathrm{~cm}^{-1}\right)$ and a long lifetime $(0.44$ $0.76 \mathrm{~ms}$ ) depending on conditions, with a luminescence that is again quenched by phosphate ions but insensitive to dissolved oxygen. A BSA-BCPDA ${ }_{39}-\mathrm{Eu}$ conjugate has been prepared, and crosslinking between the twin sulfonyl chlorides was not observed. The detection limit for free BCPDA-Eu is approximately $10 \mathrm{pmol} \mathrm{L}^{-1}$ [15] but, because most assays have relied on multiply-labeled streptavidin conjugates as a universal detection system, the actual detection limit is somewhat lower [12, 23-26]. A streptavidin-thyroglobulin ${ }_{3}-\mathrm{BCPDA}_{480}$ conjugate has also been reported [27, 28]. Although such heavily labeled conjugates provide access to lower detection limits, nonspecific binding of these large macromolecules to solid supports can present problems [44]. Measurements in these assays are typically performed in a dry well-plate using an excess of added $\mathrm{Eu}^{3+}$, meaning that contamination by exogenous $\mathrm{Eu}^{3+}$ is not an issue, which can be an advantage compared with the DELFIA system.

The Cis-Bio cryptate (Fig. 8) is a macropolycyclic ligand containing three bipyridine ligands with a dual chelatorantenna function, forming a cavity which is able to encapsulate $\mathrm{Eu}^{3+}$ and $\mathrm{Tb}^{3+}$ ions to form luminescent complexes [14]. Although this cavity results in tight metal chelation, the limitations of this system include the fact that
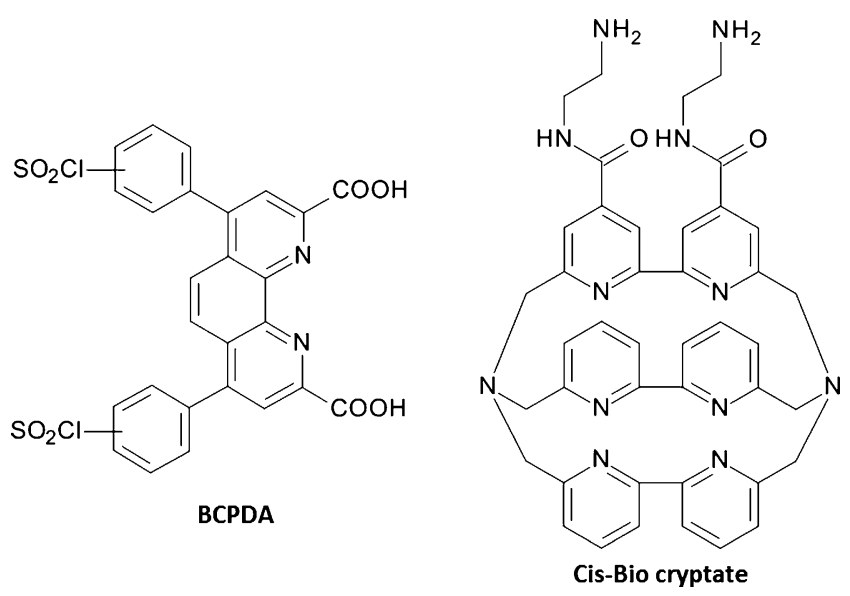

Fig. 8 Structures of BCDPA and the Cis-Bio bipyridine cryptate addition of relatively large amounts of fluoride ions is necessary to exclude water molecules, and the excitation wavelength $(307 \mathrm{~nm})$ is somewhat shorter than desired. Nevertheless, europium complexes have found application as donors in homogeneous assays, for example the timeresolved amplification of cryptate emission (TRACE) and homogeneous time-resolved fluorescence (HTRF) assays from CisBio (described in the section "Homogeneous assays") [45-47].

\section{Chelate design: overview}

A wide variety of different chelator, antenna, and proteinreactive structures are available for construction of lanthanide chelates for sensitive time-resolved luminescence assays, with the optimum system depending on the precise assay requirements. For DELFIA systems any of several non-luminescent PACs provide sufficient stability to carry the lanthanide tag through all requisite washing steps before luminescence development. Simple DTPA derivatives are generally preferred here, although luminescence development methods have also been established that enable the exploitation of the more stable DOTA derivatives. For assays requiring intrinsically luminescent chelates, this can be achieved either by simply grafting an appropriate antenna on to a PAC skeleton in the pendant chromophore strategy, or by using $\beta$-diketonate or heteroaromaticcontaining antennas in the chromophoric chelate strategy. All of these routes have been shown to be capable of leading to lanthanide chelates that have sufficient stability, luminescence, and protein reactivity for the construction of sensitive immunoassays. It is worth noting here that the brightness (i.e. the product of quantum yield and extinction coefficient) of lanthanide-based luminophores is generally low compared with those of conventional fluorophores such as fluorescein. However, this potential limitation is overcome by the effective suppression of background signals provided by the time-gated detection, giving a net effect of increased dynamic range and more sensitive assays, as will be the focus of the next section.

\section{Microtitre plate-based assays}

Heterogeneous assays

\section{Luminescence enhancement-based assays}

The most commercially successful lanthanide-based immunoassay developed to date is the dissociation-enhanced lanthanide fluorescence immunoassay (DELFIA) from Wallac Oy, which can be used in heterogeneous competitive or noncompetitive formats. In this system a non-fluorescent chelator, 
for example DTPA (discussed in the section "Non-luminescent chelates"), is used to quantitatively carry the lanthanide tag through the immunoassay and luminescence is only developed before measurement upon addition of an enhancement solution. This consists of an antenna-containing $\beta$ diketone ligand (e.g. $\beta$-naphthoyltrifluoroacetone, NTA), a synergistic agent to exclude water from the lanthanide coordination sphere (e.g. trioctylphosphine oxide, TOPO), and a non-ionic detergent (e.g. Triton X-100) in an acidic phthalate buffer [48], which causes rapid dissociation of the lanthanide from the immunocomplex and forms a strongly luminescent micellar solution complex. All four of the luminescent lanthanides ( $\mathrm{Dy}, \mathrm{Eu}, \mathrm{Sm}, \mathrm{Tb}$ ) can be utilized in the DELFIA assay, but differences in the emissive levels of the different ions necessitates use of different ligands and means that a single enhancement solution is not optimum for all ions. The detection limit for the micellar $\mathrm{Eu}^{3+}-\mathrm{NTA}$ complex (ex. $340 \mathrm{~nm}$, em. $613 \mathrm{~nm}, \Phi=0.7$ ) is of the order of $50 \mathrm{fmol} \mathrm{L}^{-1}$ [48], and $\mathrm{Eu}^{3+}$ is the lanthanide of choice for single analyte assays. This leads to protein detection limits in the low ng $\mathrm{L}^{-1}$ range (Table 1). $\mathrm{Sm}^{3+}$ also forms luminescent complexes with NTA, albeit with 70 -fold lower sensitivity (LOD $3.5 \mathrm{pmol} \mathrm{L}^{-1}$ ), and is the preferred second lanthanide for dual analyte assays [49]. For detection of three or four analytes, $\mathrm{Eu}^{3+}$ and $\mathrm{Sm}^{3+}$ luminescence can be measured in an initial step via their NTA complexes, followed by measurement of $\mathrm{Tb}^{3+}$ and $\mathrm{Dy}^{3+}$ after addition of the dipicolinic acid ligand (4-(2',4',6'-trimethoxyphenyl)pyridine-2,6-diyl)dicarboxylic acid [50]. Four analyte assays can also be constructed using cofluorescence enhancementbased systems, in which inter-chelate energy transfer occurs from nearby non-luminescent lanthanide chelates, for example $\mathrm{Gd}^{3+}$ or $\mathrm{Y}^{3+}$ [49]. Both the two-step and one-step methods deliver detection limits in the $20 \mathrm{fmol} \mathrm{L}^{-1}$ $20 \mathrm{pmol} \mathrm{L}^{-1}$ range for the four lanthanides. Three of the four luminescent lanthanides can also be efficiently sensitized by pivaloyltrifluoroacetone (LOD $0.4-20 \mathrm{pmol} \mathrm{L}^{-1}$ ) but this is an inefficient ligand for $\mathrm{Dy}^{3+}\left(\mathrm{LOD} 1 \mathrm{nmol} \mathrm{L}^{-1}\right)$.

Besides its sensitivity and capacity for multiplexing, the DELFIA system has several additional advantages, including the commercial availability of all necessary protein labeling reagents and streptavidin-lanthanide conjugates, microtitre plates, assay buffers, and enhancement solutions. The solution-based measurement also minimizes any potential discrepancies arising from surface heterogeneity. One major limitation of the DELFIA system is the loss of spatial localization that results from the dissociation step, meaning that it cannot be applied to surface-based measurements (discussed in the section "Surface-based assays"). Contamination by exogenous lanthanide is also a problem and can affect assay reproducibility [51], although the relatively high ca $1 \mathrm{nmol} \mathrm{L}^{-1} \mathrm{Eu}^{3+}$ concentration in human serum [52] should, in principle, be washed away during heterogeneous assays and not affect reproducibility. A minor practical disadvantage of the DELFIA system is the somewhat extensive washing required compared with conventional immunoassays. A typical lanthanide labeling degree is 15 25 chelates per IgG [33], and there does not seem to be any attempt in the literature to use, e.g., streptavidin-carrier protein conjugates to increase the number of lanthanide ions per immunocomplex. The same effect might also be achievable using macromolecular dendritic or polymeric chelators that could be bound to a detection antibody using the biotin-streptavidin interaction. Such a strategy could potentially dramatically increase the sensitivity of DELFIA assays by maximizing the signal intensity.

\section{Surface measurement of directly luminescent chelates}

The use of intrinsically luminescent lanthanide-containing detection reagents has several advantages compared with dissociation-enhanced solution measurements, including a reduction in the number of assay steps and washing cycles. The first such system developed was the CyberFluor FIAgen assay, which uses BCPDA-labeled (discussed in the section "Other luminescent chelates") tracers, for example, streptavidin or streptavidin-thyroglobulin conjugates as widely applicable generic detection reagents [23, 24]. Luminescence is measured on the solid phase after drying in an excess of $\mathrm{Eu}^{3+}$, which is necessary because of the limited chelate stability. An advantage of this is that the measurement is insensitive to exogenous $\mathrm{Eu}^{3+}$ contamination. Furthermore, because of the large Stokes shift and corresponding insensitivity to self-quenching, these tracers can be synthesized with surprisingly high BCPDA loadings (15 BCPDA per streptavidin or 160 BCPDA per streptavidin-thyroglobulin conjugate) resulting in improved detection limits. In fact, the luminescence emitted per luminophore seems to increase upon increased loading, such that the TG-BCPDA 160 emits luminescence equivalent to 900 free BCPDA molecules [53]. In a non-competitive heterogeneous $\alpha$-fetoprotein assay, the detection limits were improved fivefold compared with directly-labeled IgG by using streptavidin-BCPDA 15 , and fivefold again by using the streptavidin-TG-BCPDA 160 conjugate (Table 1). A macromolecular streptavidin- $\mathrm{TG}_{3.3}-\mathrm{BCPDA}_{480}$ aggregate has also been prepared and shown to afford 8 26-fold reduced detection limits for seven different analytes [27]. A more recent development has been the use of streptavidin-polyvinylamine conjugates bearing 50-100 BCPDA moieties, resulting in detection limits of $1 \mathrm{ng} \mathrm{L}^{-1}$ for PSA and a linear range spanning four orders of magnitude [54, 55]. This tracer seems to be optimum at present, although detection limits in the low $\mathrm{ng} \mathrm{L}^{-1}$ range have been reported for several proteins using the other macromolecular conjugates, and dynamic ranges for 
Table 1 Detection limits and working ranges for selected heterogeneous immunoassays utilizing lanthanide-based time-resolved luminescence

\begin{tabular}{|c|c|c|c|c|}
\hline Analyte & Reporter & Detection limit & Working range & Ref. \\
\hline \multicolumn{5}{|l|}{ DELFIA } \\
\hline Rabbit IgG & $\begin{array}{l}\text { IgG-diazophenyl-EDTA-Eu } \\
\text { IgG-SCN-Bn-EDTA-Eu }\end{array}$ & $25 \mathrm{ng} \mathrm{L}^{-1}$ & - & {$[48]$} \\
\hline Hepatitis B surface antigen & IgG-diazophenyl-EDTA-Eu & $500 \mathrm{ng} \mathrm{L}^{-1}$ & $>2$ & {$[32]$} \\
\hline Staphylococcal Enterotoxin B & Unspecified $\mathrm{IgG}-\mathrm{Eu}_{9.4}$ conjugate & $39 \mathrm{ng} \mathrm{L}^{-1}$ & $>2$ & {$[51]$} \\
\hline Yersinia pestis-specific $\mathrm{F} 1$ antigen & Unspecified $\mathrm{IgG}-\mathrm{Eu}_{6.8}$ conjugate & $24 \mathrm{ng} \mathrm{L}^{-1}$ & $>3$ & {$[51]$} \\
\hline Venezuelan equine encephalitis virus & Unspecified $\mathrm{IgG}-\mathrm{Eu}_{4.8}$ conjugate & $3.13 \times 10^{9} \mathrm{PFU} \mathrm{L}^{-1}$ & $>1$ & {$[51]$} \\
\hline Lipoprotein(a) & $\operatorname{IgG}-(\mathrm{DTTA})_{21}-\mathrm{Eu}$ & $2500000 \mathrm{ng} \mathrm{L}^{-1}$ & $>2$ & [88] \\
\hline PSA & $\mathrm{IgG}-(\mathrm{N} 1-\mathrm{ITC}-\mathrm{Eu})_{23}$ & $3.0 \mathrm{ng} \mathrm{L}^{-1}$ & $>3$ & {$[57]$} \\
\hline $\begin{array}{l}\text { Clenbuterol } \\
\text { Hydrocortisone }\end{array}$ & $\begin{array}{l}\text { IgG-DOTA-Eu } \\
\text { IgG-DOTA-Tb }\end{array}$ & $\begin{array}{l}10000 \mathrm{ng} \mathrm{L}^{-1} \\
40000 \mathrm{ng} \mathrm{L}^{-1}\end{array}$ & $\begin{array}{l}>2 \\
>1\end{array}$ & [89] \\
\hline Atrazine (comp.) & IgG-W8044-Eu & $100 \mathrm{ng} \mathrm{L}^{-1}$ & - & {$[90]$} \\
\hline \multicolumn{5}{|l|}{ FIAgen } \\
\hline Rubella IgG & $\mathrm{SA}-\mathrm{BCPDA}_{14}-\mathrm{Eu}$ & $10000 \mathrm{IU} \mathrm{L}^{-1}$ & - & {$[28]$} \\
\hline Thyroxine-binding globulin & SA-BCPDA-Eu & $400 \mu \mathrm{g} \mathrm{L}^{-1}$ & $>2$ & {$[26]$} \\
\hline$\alpha$-fetoprotein & IgG-BCPDA-Eu & $5000 \mathrm{ng} \mathrm{L}^{-1}$ & - & {$[53]$} \\
\hline$\alpha$-fetoprotein & SA-BCPDA $14-E u$ & $1000 \mathrm{ng} \mathrm{L}^{-1}$ & - & [53] \\
\hline$\alpha$-fetoprotein & SA-TG-BCPDA $160-E u$ & $200 \mathrm{ng} \mathrm{L}^{-1}$ & - & {$[53]$} \\
\hline Choriogonadotrophin & SA-BCPDA-Eu & $1 \mathrm{IU} \mathrm{L}^{-1}$ & $>2$ & {$[24]$} \\
\hline Human pancreatic isoamylase & SA-TG-BCPDA-Eu & $1100 \mathrm{ng} \mathrm{L}^{-1}$ & $>2$ & [91] \\
\hline Growth hormone & $\mathrm{SA}-\mathrm{TG}_{3.3}-\mathrm{BCPDA}_{480}-\mathrm{Eu}$ & $3 \mathrm{ng} \mathrm{L}^{-1}$ & - & [27] \\
\hline Carcinoembryonic antigen & $\mathrm{SA}-\mathrm{TG}_{3.3}-\mathrm{BCPDA}_{480}-\mathrm{Eu}$ & $11 \mathrm{ng} \mathrm{L}^{-1}$ & - & {$[27]$} \\
\hline PSA & SA-B-PVA-BCPDA & $1 \mathrm{ng} \mathrm{L}^{-1}$ & 4 & {$[54,55]$} \\
\hline Cortisol (comp.) & $\mathrm{SA}-\mathrm{BCPDA}_{15}-\mathrm{Eu}$ & $10000 \mathrm{ng} \mathrm{L}^{-1}$ & $>1$ & {$[25]$} \\
\hline Digoxin (comp.) & SA-TG-BCPDA $150-E u$ & $250 \mathrm{ng} \mathrm{L}^{-1}$ & $>1$ & [92] \\
\hline \multicolumn{5}{|l|}{$\beta$-diketones } \\
\hline$\alpha$-fetoprotein & SA-BHHCT $21-\mathrm{Eu}$ & $4.1 \mathrm{pg} \mathrm{L}^{-1}$ & - & {$[41]$} \\
\hline Thyroid-stimulating hormone & SA-BSA ${ }_{1.8}-\mathrm{BTBCT}_{77}-\mathrm{Eu}$ & $0.1 \mathrm{mIU} \mathrm{L}^{-1}$ & $>3$ & [42] \\
\hline $\mathrm{T} 4$ (comp.) & T4-BSA-BTBCT $23-\mathrm{Eu}$ & $5.8 \mathrm{nmol} \mathrm{L}^{-1}$ & - & {$[42]$} \\
\hline T4 (comp.) & T4-BSA-B, SA-BCTOT-Eu & $6.7 \mathrm{nmol} \mathrm{L}^{-1}$ & - & {$[40]$} \\
\hline \multicolumn{5}{|l|}{ Nanoparticle-based } \\
\hline PSA & SA-coated $\mathrm{Eu}(\beta-\mathrm{NTA})_{3}$-doped polystyrene NPs & $1.6 \mathrm{ng} \mathrm{L}^{-1}$ & 4 & {$[56]$} \\
\hline PSA & IgG-coated $\mathrm{Eu}(\beta-\mathrm{NTA})_{3}$-doped polystyrene NPs & $0.21 \mathrm{ng} \mathrm{L}^{-1}$ & 4 & [57] \\
\hline Adenovirus & IgG-coated $\mathrm{Eu}(\beta-\mathrm{NTA})_{3}$-doped polystyrene NPs & $\begin{array}{l}5650000 \text { virus } \\
\text { particles } L^{-1}\end{array}$ & 4 & {$[58]$} \\
\hline Hepatitis B surface antigen (HBsAg) & $\operatorname{IgG}$-coated $\mathrm{Eu}(\beta-\mathrm{NTA})_{3}$-doped polystyrene NPs & $28 \mathrm{ng} \mathrm{L}^{-1}$ & 4 & {$[59]$} \\
\hline Listeria spp. & IgG-coated $\mathrm{Eu}(\beta-\mathrm{NTA})_{3}$-doped polystyrene NPs & $20000 \mathrm{CFU} \mathrm{L}^{-1}$ & 4 & {$[60]$} \\
\hline Anthrax PA & SA-coated polystyrene NPs, biotinylated anti-SA, SA-Eu & $10 \mathrm{ng} \mathrm{L}^{-1}$ & 4 & {$[61]$} \\
\hline $\begin{array}{l}\text { Hepatitis B surface antigen (HBsAg) } \\
\text { Anti-HIV-1 IgG }\end{array}$ & $\begin{array}{l}\mathrm{F}(\mathrm{ab})_{2} \text {-coated } \mathrm{Eu}(\beta-\mathrm{NTA})_{3} \text {-doped polystyrene NPs } \\
\text { Pyridine-based Tb chelate-labeled HIV-1 antigen }\end{array}$ & $\begin{array}{l}0.013 \mathrm{ng} \mathrm{L}^{-1} \\
\text { n.d. }\end{array}$ & $\begin{array}{l}4 \\
-\end{array}$ & {$[62]$} \\
\hline \multicolumn{5}{|c|}{ Enzyme-amplified lanthanide luminescence } \\
\hline$\alpha$-fetoprotein & $\begin{array}{l}\text { IgG-ALP } \\
\text { Salicylphosphate/salicylic acid/Tb-EDTA }\end{array}$ & $1.4 \mathrm{ng} \mathrm{L}^{-1}$ & $>2$ & {$[67]$} \\
\hline$\alpha$-fetoprotein & $\begin{array}{l}\text { Bt-pAb/SA-ALP } \\
\text { 5-fluorosalicyl phosphate/5-fluorosalicylic acid/Tb-EDTA }\end{array}$ & $0.15 \mathrm{ng} \mathrm{L}^{-1}$ & $>2$ & {$[68]$} \\
\hline$\alpha$-fetoprotein & $\begin{array}{l}\text { IgG-ALP } \\
\text { Diflunisal phosphate/diflunisal/Tb-EDTA }\end{array}$ & $3 \mathrm{ng} \mathrm{L}^{-1}$ & $>2$ & {$[66]$} \\
\hline Interleukin 6 & $\begin{array}{l}\text { SA-ALP } \\
\text { Diflunisal phosphate/diflunisal/Tb-EDTA }\end{array}$ & $0.5 \mathrm{ng} \mathrm{L}^{-1}$ (serum) & - & {$[69]$} \\
\hline PSA & $\begin{array}{l}\text { SA-ALP } \\
\text { Diflunisal phosphate/diflunisal/Tb-EDTA }\end{array}$ & $\begin{array}{l}2 \mathrm{ng} \mathrm{L}^{-1} \\
10 \mathrm{ng} \mathrm{L}^{-1} \text { (serum) }\end{array}$ & - & [70] \\
\hline
\end{tabular}

comp. $=$ competitive 
BCPDA-based assays are generally in the range of 2-3 orders of magnitude (Table 1).

Several of the $\beta$-diketone ligands (discussed in the section " $\beta$-Diketone-based luminescent chelates") have also been used in immunoassays. Streptavidin-BHHCT $\mathrm{B}_{21}$ has been developed as a generic detection reagent for luminescence measurement on either the solid phase or in solution after dissolution of the immunocomplex from the well plate [41]. For $\alpha$-fetoprotein solid and solution phase detection limits of $46 \mathrm{pg} \mathrm{L}^{-1}$ and $4.1 \mathrm{pg} \mathrm{L}^{-1}$, respectively, were obtained (Table 1), which are at least four orders of magnitude lower than the aforementioned BCPDA-based assay and also superior to previous $\alpha$-fetoprotein assays. A streptavidin-BSA ${ }_{1.8}-\mathrm{BTBCT}_{77}$ conjugate has also been prepared and used for zeptomole detection of hTSH in a sandwich assay and found to give results comparable with those from a DELFIA-based system [42]. Two competitive assays for serum T4 have also been developed using T4BSA-BTBCT ${ }_{23}$ or T4-BSA-biotin/streptavidin-BCTOT as competing analytes to give low $\mathrm{nmol} \mathrm{L} \mathrm{L}^{-1}$ detection limits, which is sufficient for clinical diagnosis [40, 42].

Intrinsically luminescent lanthanide chelate-labeled detection reagents therefore provide an effective means of increasing sensitivity and linear range in immunoassays for a variety of different analytes. Performing luminescence measurements on a well plate surface necessitates a sufficient amount of tracer being present in the immunocomplex, and a greater number of lanthanide tags can typically be installed in biomolecules than conventional fluorophores, because of the lack of self-quenching. The number of lanthanide ions per immunocomplex can be increased even further by using extensively labeled proteins or other carrier macromolecules, although this has yet to be exploited for other dyes besides BCPDA and may be an attractive means of increasing sensitivity in other assays.

\section{Nanoparticle-based assays}

An interesting alternative to labeling an antibody or generic detection reagent with small-molecule lanthanide chelates is, instead, to incorporate the luminescent chelates inside a nanoparticle and coat the surface with the biomolecule. This method has resulted in broad linear ranges over, generally, four orders of magnitude and demonstrates the usefulness of time-resolved luminescence measurement especially in the field of protein detection. For example, with a typical 107-nm polystyrene bead containing $>30,000$ $\mathrm{Eu}(\mathrm{III})$-2-thenoyltrifluoroacetone complexes, a much higher lanthanide-to-biomolecule ratio can be achieved than by conventional methods. The potential of streptavidin-coated luminescent nanoparticles was demonstrated in a PSA assay, giving zeptomole $\left(10^{-21} \mathrm{~mol}\right)$ sensitivity and a linear range spanning four orders of magnitude (Table 1), a 100-fold increase over conventional methods using Eu-labeled streptavidin [56]. A modest increase in sensitivity was observed by using PSAspecific IgG-coated NPs (Table 1) [57]. IgG-coated nanoparticles have also been used for detection of an adenovirus, hepatitis B virus (HBsAg) and even bacteria (Table 1) [58-60]. An interesting signal amplification method has also been reported in a sandwich assay for anthrax toxin, in which a biotinylated secondary antibody is first bound to a streptavidin-coated luminescent nanoparticle before forming a macromolecular complex using biotinylated antistreptavidin and streptavidin-Eu chelates [61].

Although these studies demonstrate the potential of lanthanide-doped nanoparticles, an interesting future development that has not been fully exploited is that of multicolor assays utilizing several different lanthanides. A dual analyte assay for hepatitis B surface antigen (HBsAg) and human immunodeficiency virus 1 (HIV-1) antibodies has been developed using $\mathrm{F}(\mathrm{ab})_{2}$-coated $\mathrm{Eu}$ nanoparticles and a small molecule pyridine-based $\mathrm{Tb}$ chelate-labeled antigen [62]. In a move toward an entirely nanoparticle-based multiplexing assay, the synthesis of 45$\mathrm{nm}$ poly(styrene-acrylic acid) nanoparticles containing each of the four luminescent lanthanides with appropriate sensitizers $(>1,000$ chelates per nanoparticle) has been reported and applied to an immunoassay to give detection limits for PSA of 1.6, 2.4, 10.1, and $114.2 \mathrm{ng} \mathrm{L}^{-1}$ for the $\mathrm{Eu}^{3+}, \mathrm{Tb}^{3+}, \mathrm{Sm}^{3+}$ and $\mathrm{Dy}^{3+}$-doped nanoparticles, respectively [63]. One reason for the limited application of polystyrene nanoparticles may be the less-than-optimum physical properties of the polymer itself, which include larger particle size, swelling, and leakage of the encapsulated molecules through surface defects [64]. A duallanthanide immunoassay for human and mouse IgG using $40 \mathrm{~nm}$ Eu-doped and Sm-doped silica nanoparticles has recently appeared but these multiplexing techniques have yet to be fully exploited [65]. These silica nanoparticles have several advantages over latex-based ones, including improved aqueous solubility and coupling chemistry, because of the silanol surface [64].

\section{Enzyme-amplified lanthanide luminescence (EALL)}

EALL assays utilize an enzyme as label, which converts a substrate into a product which is sufficiently different in terms of its chelating or spectroscopic properties to enable distinction between the two. The key advantage of this technique compared with the hitherto discussed assay formats is the enzymatic amplification step, although substrate conversion itself necessitates an additional assay step. Still, the potential for highly sensitive assays based on EALL is certainly one of the most promising in the field. 
Most such assays use alkaline phosphatase (ALP) conjugates to convert salicylic acid phosphates into the corresponding salicylic acid derivative, the latter of which forms a luminescent complex with $\mathrm{Tb}^{3+}$ and EDTA. The choice of substrate (Fig. 9, X substituent) depends on the assay requirements, with more soluble substrates being preferred for microtitre plate assays and more hydrophobic substrates being preferred for surface-based assays where signal localization is desired (discussed in the section "Surface-based assays"). The X substituent also affects the final luminescence properties of the chelate, and a comparison of 14 different ligands revealed that diflunisal $\left(X=5-\left(2^{\prime}, 4^{\prime}\right.\right.$-difluorophenyl)) gave amongst the most intense signals [66]. The limit of detection for ALP has been reported as 0.2 amol [44], and the use of diflunisal and related fluorinated substrates has led to detection limits in the low $\mathrm{ng} \mathrm{L}^{-1}$ range for analytes such as $\alpha$-fetoprotein, interleukin 6, and PSA, even in serum (Table 1) [66-70]. These detection limits are at least equal to those using the luminophore-labeled tracers discussed earlier and confirm the value of EALL systems, especially when using widely available antibody-ALP conjugates.

Besides the ALP-based assays, xanthine oxidase-salicylaldehyde and $\beta$-galactosidase-salicyl- $\beta$-D-galactoside systems can also be used to generate salicylic acid- $\mathrm{Tb}$ (III)EDTA complexes in solution [44]. These systems have seldom been used, however, perhaps because of their reduced sensitivity and the widespread availability of
antibody-ALP conjugates. Hemin-mediated dimerization of $p$-hydroxybenzoic acid in the presence of $\mathrm{H}_{2} \mathrm{O}_{2}$ also generates a species capable of sensitizing $\mathrm{Tb}$ (III)-EDTA, although this has not yet been applied to an immunoassay format [71]. EALL systems have yet to be extensively developed for lanthanides other than $\mathrm{Tb}^{3+}$, although glucose oxidase-mediated hydrolysis of 1,10-phenanthroline-2,9-dicarboxylic acid dihydrazide to the corresponding diacid has been shown to sensitize $\mathrm{Eu}^{3+}$ [44]. One future objective in this area is the continued development of EALL systems which operate at neutral $\mathrm{pH}$ rather than the strongly alkaline systems above which require addition of EDTA to avoid precipitation of the lanthanide hydroxide, because the EDTA itself sensitizes lanthanides to a small extent and contributes to the background signal.

\section{Homogeneous assays}

Homogeneous immunoassays are potentially the most convenient assay format, in principle requiring only addition of the sample to the premixed reagents. Timeresolved luminescence can be extremely beneficial for such assays, because the inherently high background signals can be minimized. However, sensitive assays are much more difficult to achieve than with heterogeneous systems. Homogeneous assays are generally based on some form of LRET system, in which the presence of antigen either displaces a donor or acceptor-labeled antigen analogue
Fig. 9 Enzyme-amplified lanthanide luminescence. Top: enzyme-mediated conversion of a substrate into a product that forms luminescent chelates with lanthanides. Bottom: conversion of salicylic acid phosphate derivatives by ALP affords the corresponding salicylic acid which forms luminescent chelates with Tb-EDTA

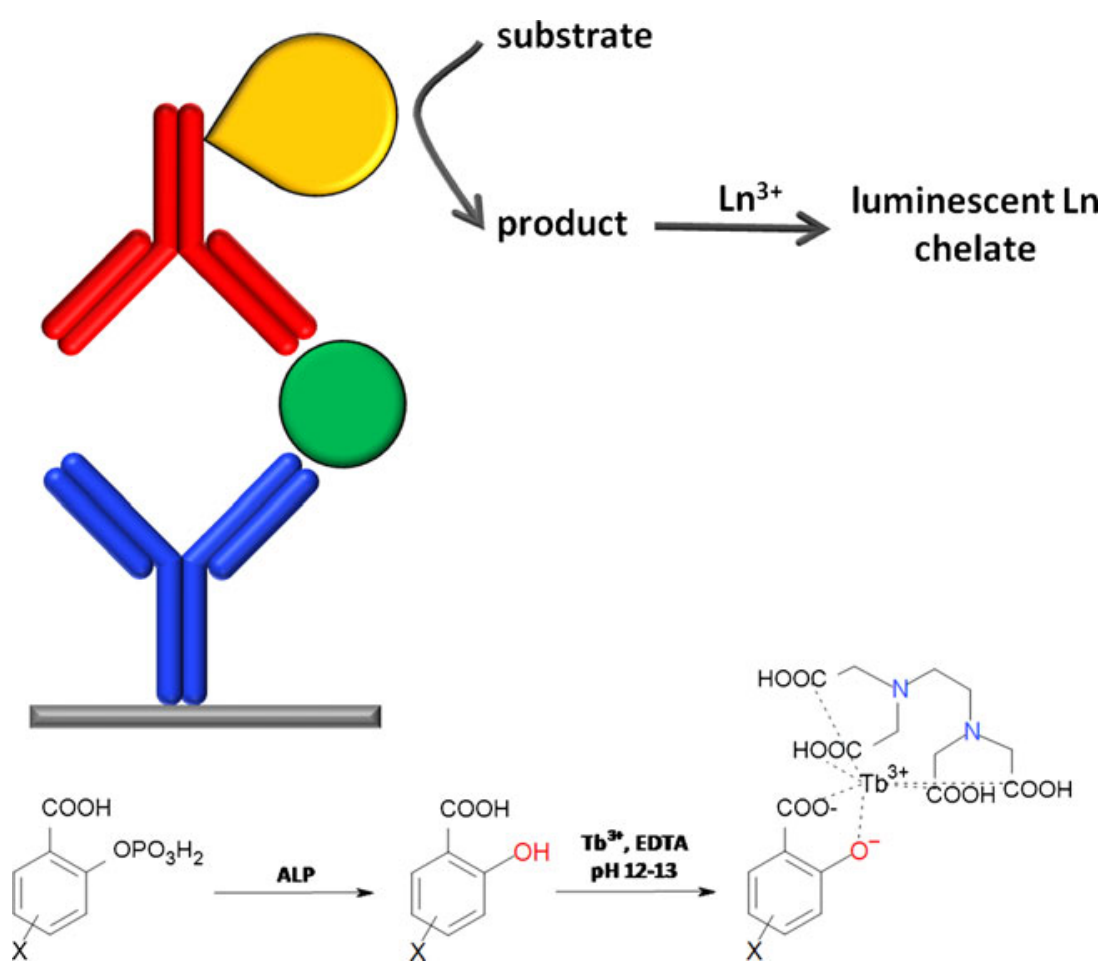


from an acceptor or donor-labeled antibody (causing LRET to decrease in response to antigen concentration), or bridges two labeled antibodies (causing LRET to occur in response to antigen concentration; Fig. 1).

The most common system is the time-resolved amplification by cryptate emission (TRACE; also known as homogeneous time-resolved fluorescence, HTRF) system developed by Cisbio International, which relies on a europium cryptate label (Fig. 8) as donor and a crosslinked allophycocyanin (XL665) as acceptor [45]. One surprising benefit of this system is that the efficient LRET between these two species actually results in a signal increase compared with radiative deactivation of the isolated $\mathrm{Eu}$ cryptate [46]. This system was first developed for an assay for prolactin in which two IgGs were separately labeled with the cryptate and XL665 [45]. In the presence of analyte, $337 \mathrm{~nm}$ excitation of the cryptate is followed by energy transfer to the XL665 which ultimately emits at $665 \mathrm{~nm}$ with a lifetime of $0.25 \mathrm{~ms}$ and can be measured and compared with the reference $620 \mathrm{~nm}$ cryptate emission for unbound $\mathrm{IgG}$, resulting in a detection limit of $0.3 \mu \mathrm{g} \mathrm{\textrm {L } ^ { - 1 }}$ prolactin (Table 2). A detection limit of $<600 \mathrm{ng} \mathrm{L}^{-1}$ for interleukin-13 has also been obtained [47].

Small-molecule acceptors can also be used in LRETbased systems. In a competitive assay for urinary albumin, albumin-Cy5 was used as a tracer to accept energy from the pyridine-based luminescent donor ITC-TEKES-Eu, giving a rather high detection limit in the $\mathrm{mg} \mathrm{L}^{-1}$ range [72]. The working range of this assay was nonetheless sufficient for diagnosis of microalbuminuria.

Antibody Fab fragment-coated $\mathrm{Eu}^{3+}$ chelate-doped $92 \mathrm{~nm}$ nanoparticles have been used in conjunction with an estradiol-AlexaFluor 680 acceptor to detect pmol L $\mathrm{L}^{-1}$ levels of estradiol in a competitive assay [73]. Sensitivity in this assay was increased 20-fold compared with assays using soluble chelates [74]. Nanoparticle-based donors and acceptors have also been combined in a PSA assay in which anti-PSA antibodies on the two surfaces bring together the donor and acceptor nanoparticles and lead to a detection limit of $0.1 \mu \mathrm{g} \mathrm{L}^{-1}$ [75].

The key benefit of using lanthanide complexes in homogeneous assays arises from the fact that such assays are ideally performed by direct addition of the biological sample to the premixed assay reagents. In comparison with heterogeneous assays in which background interferences can be removed by multiple washing steps, these species are still present during measurement in a homogeneous assay, and the use of lanthanide chelates and time-gated acquisition enables the background signal to be efficiently removed.

\section{Surface-based assays}

Despite the proven benefits of using lanthanide chelates in microtitre plate-based assays, the extension of timeresolved luminescence techniques to surface-based assays has been less widespread. The ability to use lanthanide chelates on surfaces used in key proteomics techniques, for example membranes, gels, and tissue slices, would be a major breakthrough, because these materials typically have high autofluorescence backgrounds which could be removed by using time-resolved techniques. However, a lack of suitable instrumentation has hindered the development of these techniques. This is especially true for the time-resolved luminescence measurement of polyacrylamide gels, nitrocellulose, or PVDF membranes to enable application of gel-based protein analysis systems, for example immunoblot and SDS-PAGE. To the best of our knowledge, only one instrument for time-resolved fluorescence measurement of gels and membranes is currently commercially available (SpectraMax Paradigm, Molecular Devices). This prototype shows the high potential of TRL measurements in gel-based analysis systems [17]. Interestingly, a linear range of five orders of magnitude was reported for this instrument, demonstrating the high potential of time-resolved luminescence for immunoblot applications.

The advantages of time-resolved luminescence detection for microscopy studies, especially for highly autofluorescent tissue materials, are obvious. Surface-based assays require intrinsically luminescent lanthanide chelates, because spatial resolution would be lost in a dissociationenhanced format. Although several europium-based stains for visualization of tissue slices and membranes have been
Table 2 Detection limits and working ranges for selected homogeneous immunoassays utilizing lanthanide-based time-resolved luminescence

\begin{tabular}{lllll}
\hline Analyte & Reporters & Detection limit & Working range & Ref. \\
\hline Prolactin & $\begin{array}{l}\text { Donor: IgG-TBP-Eu } \\
\text { Acceptor: IgG-APC } \\
\text { Donor: IgG-Eu cryptate }\end{array}$ & $300 \mathrm{ng} \mathrm{L}^{-1}$ & - & [45] \\
Acceptor: IgG-biotin-SA-XL665 & $<600 \mathrm{ng} \mathrm{L}^{-1}$ & - & [47] \\
Albumin (comp.) & $\begin{array}{l}\text { Donor: IgG-TEKES-ITC } 2.7-\mathrm{Eu} \\
\text { Acceptor: albumin-Cy5 }\end{array}$ & $5.5 \mathrm{mg} \mathrm{L}^{-1}$ & $>1$ & [72] \\
Estradiol (comp.) & $\begin{array}{l}\text { Donor: Fab-coated-Eu-chelate NP } \\
\text { Acceptor: estradiol-Alexa } 680\end{array}$ & $70 \mathrm{pmol} \mathrm{L}^{-1}$ & - & [73] \\
& & & & \\
\hline
\end{tabular}


developed since the 1960s [76, 77], the first lanthanidelabeled immunoreagents for dedicated specific surfacebased detection did not appear until 1988 [5]. In this case, anti-rabbit IgG was labeled with a europium-W1014 chelate (structure unspecified) and used for immunohistochemical staining using a conventional fluorescence microscope. The use of dehydrated histological sections was necessary because of aqueous quenching, and both intensity and stability under steady-state excitation were inferior to those of fluorescein-labeled IgG.

Full exploitation of the potential of lanthanide-labeled biomolecules required the development of a luminescence microscope capable of time-gated detection; this was first applied to lanthanide-labeled biomolecules in 1990 [78]. These early experiments demonstrated that 100-300 nm milled $\mathrm{Eu}^{\mathrm{III}}$-doped yttrium oxysulfide polycarboxylic acidstabilized nanoparticle phosphors coated with $\mathrm{IgG}$ or avidin could successfully bind corresponding targets on latex beads and cell surfaces. A similar prototype instrument was developed and used for immunohistochemical analysis of various specimens after staining with antisera and streptavidin coupled to a pyridine-based europium chelate, enabling efficient removal of background luminescence and equal sensitivity to fluorescein-labeled and enzyme-labeled antibodies [79]. Labeling of antibodies with the stable $\mathrm{Eu}-$ W8044 chelate (structure unspecified) has also been found to reduce the autofluorescence of glutaraldehyde-fixed colon cancer cells and enable signal-to-background ratios of 2400:1 to be obtained [80]. Likewise, Eu-terpyridinelabeled antibodies have been shown to improve signal-tobackground ratios in islet cells by a factor of 12 compared with conventional fluorescein-labeled antibodies [81]. Similarly, a Eu-terpyridine chelate has been attached to anti-PSA antibodies and bound to prostate cells [82]. Several $\beta$-diketonate dyes have also been used for microscopy studies. Antibodies labeled with BPPCT (Fig. 7) have been used for detection of cysts of the water-borne pathogen Giardia lamblia on filter membranes, resulting in a 30 -fold increase in contrast relative to conventional immunoreagents [83]. Giardia and Cryptosporidium have also been detected by use of BHHSTlabeled antibodies, which resulted in tenfold enhancement of signal-to-noise ratio [43, 84]. It has also been shown recently that the luminescence of surface-based BHHCT complexes is enhanced in the presence of silver nanostructures, leading to twofold increased brightness during detection of G. lamblia cells [85]. These techniques enable sensitive detection of pathogens in environmental water samples containing multiple autofluorescent species, for example algae, mineral particles, and organic debris.

For generic detection reagents, streptavidin-thyroglobulin-BCPDA (discussed in the section "Surface measurement of directly luminescent chelates") has been used for analysis of tissue-bound biotinylated anti-myosin antibodies, and its photostability has been reported to be superior to that of previous chelates [86]. Streptavidin-coated Eu-containing nanoparticles have also been used, enabling detection of single nanoparticles using a TRL microscope [82]. In model studies using biotinylated PSA, a linear range of two orders of magnitude was reported, although the authors note that biological variation is expected to constitute the major source of error when attempting to quantify antigens in tissue sections. An unspecified streptavidin-Eu chelate has also been used for detection of biotin-tyramide after enzymatic deposition by an antibody-peroxidase conjugate, enabling signal amplification [87].

Considering all results described for surface-based assays, it is clear that this area is an ideal field of application for time resolved luminescence. However, few instruments are currently able to fully exploit this possibility.

\section{Conclusions}

The objective of this review has been to explain the benefits of lanthanide-based time-resolved luminescence measurements in immunoassays. Using time-gated acquisition the background signal can be efficiently suppressed, enabling more sensitive detection of proteins using lanthanide chelate tags than is possible with chromophore-based or conventional fluorophore-based labels. This ultimately leads to lower detection limits and wider linear ranges for the analyte in question. This is especially valuable for protein detection where these criteria are of particular importance and where high autofluorescence is normally observed when time-gated detection is not used.

Although it is difficult to reliably compare sensitivities reported for different lanthanide chelates obtained for different analytes and assay configurations in different laboratories, the heterogeneous DELFIA, FIAgen, nanoparticle, and EALL-based assays are all capable of delivering sensitivity in the lower $n g \mathrm{~L}^{-1}$ range. For the DELFIA system this is derived from an intensely luminescent solution complex, whereas the other assays rely either on the extensive incorporation of lanthanides into a multiprotein complex or nanoparticle, or on enzymatic amplification to increase the number of detectable labels per immunocomplex. It would be interesting, therefore, to investigate whether the sensitivity of the DELFIA system could be increased even further by use of macromolecular chelates that incorporate hundreds of lanthanide ions rather than the 15-25 ions used with single antibodies. The sensitivity of the EALL assays could also, perhaps, be improved via more extensive testing of enzyme-substrate pairs than the predominantly ALP-salicylic acid-based systems that have been used to date. For the homogeneous 
assays that have been developed the sensitivity is typically in the high $n g \mathrm{~L}^{-1}$ range, so these are more suitable for applications where speed is more important than sensitivity.

A key advantage of lanthanide time-resolved luminescencebased assays is the increased linear range because of suppression of the background signal, and many of the heterogeneous assays discussed above are able, in principle, to provide linear ranges spanning five orders of magnitude. However, for the actual assays discussed here, linear ranges of 2-3 orders of magnitude are typical for the DELFIA, FIAgen, $\beta$-diketonate, and EALL-based assays, with nanoparticlebased assays consistently delivering linear ranges spanning at least four orders of magnitude. With the difference between high abundance and low abundance proteins spanning an estimated twelve orders of magnitude [1], it is clear that further improvements in instrumentation and assay development are desirable here. In conclusion, more emphasis should be given to the use of time-resolved luminescence as an ideal tool to meet the unique challenges of the field of protein detection, for example broad linear ranges, high sensitivity, and excellent signal-to-background ratios.

Acknowledgments The authors gratefully acknowledge the helpful comments on this manuscript of Professor Ralf Hoffmann. This work was supported by BMBF Innoprofile, project number 03IP604.

Open Access This article is distributed under the terms of the Creative Commons Attribution Noncommercial License which permits any noncommercial use, distribution, and reproduction in any medium, provided the original author(s) and source are credited.

\section{References}

1. Corthals G, Wasinger V, Hochstrasser D, Sanchez J-C (2000) The dynamic range of protein expression: a challenge for proteomic research. Electrophoresis 21:1104-1115

2. Weissman S (1942) Intramolecular energy transfer: the fluorescence of complexes of europium. J Chem Phys 10:214-217

3. Hemmilä I, Laitala V (2005) Progress in lanthanides as luminescent probes. J Fluoresc 15(4):529-542

4. Aubin J (1979) Autofluorescence of viable cultured mammalian cells. J Histochem Cytochem 27(1):36-43

5. Soini E, Pelliniemi L, Hemmilä I, Mukkala V-M, Kankare J, Fröjdman K (1988) Lanthanide chelates as new fluorochrome labels for cytochemistry. J Histochem Cytochem 36(11):14491451

6. Soini E, Hemmilä I (1979) Fluoroimmunoassay: present status and key problems. Clin Chem 25(3):353-361

7. Eliseeva S, Bünzli J-C (2010) Lanthanide luminescence for functional materials and bio-sciences. Chem Soc Rev 39(1):189227

8. Karraker D (1970) Coordination of trivalent lanthanide ions. J Chem Educ 47(6):424-430

9. Cacheris W, Nickle S, Sherry A (1987) Thermodynamic study of lanthanide complexes of 1,4,7-Triazacyclononane- $N, N^{\prime}, N^{\prime \prime}$-triacetic Acid and 1,4,7,10-Tetraazacyclododecane- $N, N^{\prime}, N^{\prime \prime}, N^{\prime \prime \prime}$-tetraacetic Acid. Inorg Chem 26:960-962
10. Binnemans K (2005) Rare-earth beta-diketonates. In: Gschneidner K Jr, Bünzli J-C, Pecharsky V (eds) Handbook on the physics and chemistry of rare earths, vol 35. Elsevier B. V., pp 107-272

11. Yamada S, Miyoshi F, Kano K, Ogawa T (1981) Highly sensitive laser fluorimetry of europium(III) with 1,1,1-trifluoro-4-(2-thienyl)2,4-butanedione. Anal Chim Acta 127:195-198

12. Diamandis E (1988) Immunoassays with time-resolved fluorescence spectroscopy: principles and applications. Clin Biochem 21:139-150

13. Moore E, Samuel A, Raymond K (2009) From antenna to assay: lessons learned in lanthanide luminescence. Acc Chem Res 42 (4):542-552

14. Alpha B, Lehn J-M, Mathis G (1987) Energy transfer luminescence of europium(III) and Terbium(III) cryptates of macrobicyclic polypyridine ligands. Angew Chem Int Ed Engl 26(3):266-267

15. Evangelista R, Pollak A, Allore B, Templeton E, Morton R, Diamandis E (1988) A new europium chelate for protein labelling and time-resolved fluorometric applications. Clin Biochem 21:173-178

16. Selvin P, Rana T, Hearst J (1994) Luminescence resonance energy transfer. J Am Chem Soc 116(13):6029-6030

17. Zuchner T, Schumer F, Berger-Hoffmann R, Müller K, Lukas M, Zeckert K, Marx J, Hennig H, Hoffmann R (2009) Highly sensitive protein detection based on lanthanide chelates with antenna ligands providing a linear range of five orders of magnitude. Anal Chem 81(22):9449-9453

18. Armelao L, Quici S, Barigelletti F, Accorsi G, Bottaro G, Cavazzini M, Tondello E (2010) Design of luminescent lanthanide complexes: from molecules to highly efficient photo-emitting materials. Coord Chem Rev 254:487-505

19. Cross J, Dadabhoy A, Sammes P (2004) The sensitivity of the lehn cryptand-europium and terbium (III) complexes to anions compared to a coordinatively saturated systems. J Lumin 110 (3):113-124

20. Sabbatini N, Guardigli M, Lehn J-M (1993) Luminescent lanthanide complexes as photochemical supramolecular devices. Coord Chem Rev 123:201-228

21. Latva M, Takalo H, Mukkala V-M, Matachescu C, RodríguezUbis J, Kankare J (1997) Correlation between the lowest triplet state energy level of the ligand and lanthanide(III) luminescence quantum yield. J Lumin 75:149-169

22. Perkin Elmer product manual for DELFIA(R) Eu-Labelling Reagent 1244-301 (2000) http://shop.perkinelmer.com/content/ Manuals/man 1244-301.pdf. Accessed 5 May 2011

23. Chan M, Bellem A, Diamandis E (1987) Time-resolved immunofluorometric assay of alpha-fetoprotein in serum and amniotic fluid, with a novel detection system. Clin Chem 33 (11):2000-2003

24. Khosravi M, Diamandis E (1987) Immunofluorometry of choriogonadotropin by time-resolved fluorescence spectroscopy, with a new europium chelate as label. Clin Chem 33(11):1994-1999

25. Diamandis E, Bhayana V, Conway K, Reichstein E, Papanastasiou-Diamandis A (1988) Time-resolved fluoroimmunoassay of cortisol in serum with a europium chelate as label. Clin Biochem 21:291-296

26. Tan Y, Khosravi M, Diamandis E (1989) Time-resolved immunofluorometric assay for thyroxine-binding globulin in serum. J Immunoassay 10(4):413-428

27. Morton R, Diamandis E (1990) Streptavidin-based macromolecular complex labeled with a europium chelator suitable for time-resolved fluorescence immunoassay applications. Anal Chem 62(17):18411845

28. Shankaran P, Reichstein E, Khosravi M, Diamandis E (1990) Detection of immunoglobulins $G$ and $M$ to rubella virus by 
time-resolved immunofluorometry. J Clin Microbiol 28(3):573579

29. Härmä H, Soukka T, Lövgren T (2001) Europium nanoparticles and time-resolved fluorescence for ultrasensitive detection of prostate-specific antigen. Clin Chem 47(3):561-568

30. Soukka T, Paukkunen J, Härmä H, Lönnberg S, Lindroos H, Lövgren $\mathrm{T}$ (2001) Supersensitive time-resolved immunofluorometric assay of free prostate-specific antigen with nanoparticle label technology. Clin Chem 47(7):1269-1278

31. Wieder I (1978) Background rejection in fluorescence immunoassay. In: Knapp W, Holubar K, Wick G (eds) Immunofluorescence and related staining techniques. Proc VIth International Conference on Immunofluorescence and Related Staining Techniques, Vienna, pp $67-80$

32. Siitari H, Hemmilä I, Soini E, Lövgren T, Koistinen V (1983) Detection of hepatitis B surface antigen using time-resolved fluoroimmunoassay. Nature 301:258-260

33. Mukkala V-M, Mikola H, Hemmilä I (1989) The synthesis and use of activated $\mathrm{N}$-benzyl derivatives of diethylenetriaminetetraacetic acids: alternative reagents for labeling of antibodies with metal ions. Anal Biochem 176:319-325

34. De Silva C, Vagner J, Lynch R, Gillies R, Hruby V (2010) Optimization of time-resolved fluorescence assay for detection of europium-tetraazacyclododecyltetraacetic acid-labeled ligand-receptor interactions. Anal Biochem 398:15-23

35. Li M, Selvin P (1995) Luminescent polyaminocarboxylate chelates of terbium and europium: the effect of chelate structure. J Am Chem Soc 117(31):8132-8138

36. Li M, Selvin P (1997) Amine-reactive forms of a luminescent diethylenetriaminepentaacetic acid chelate of terbium and europium: attachment to dna and energy transfer measurements. Bioconjug Chem 8(2):127-132

37. Chen J, Selvin P (1999) Thiol-reactive luminescent chelates of terbium and europium. Bioconjug Chem 10(2):311-315

38. Yuan J, Matsumoto K (1996) Synthesis of a new tetradentate $\beta$ diketonate-europium chelate that can be covalently bound to proteins in time-resolved fluorometry. Anal Sci 12:695-699

39. Yuan J, Matsumoto K (1997) Synthesis of a new tetradentate $\beta$-diketonate-europium chelate and its application for timeresolved fluorimetry of albumin. J Pharm Biomed Anal 15:1397-1403

40. Wu F-B, Han S-Q, Zhang C, He Y-F (2002) Synthesis of a highly fluorescent $\beta$-diketone-europium chelate and its utility in timeresolved fluoroimmunoassay of serum total thyroxine. Anal Chem 74(22):5882-5889

41. Yuan J, Matsumoto K, Kimura H (1998) A new tetradentate $\beta$ diketonate-europium chelate that can be covalently bound to proteins for time-resolved fluoroimmunoassay. Anal Chem 70 (3):596-601

42. Wu F-B, Zhang C (2002) A new europium $\beta$-diketone chelate for ultrasensitive time-resolved fluorescence immunoassays. Anal Biochem 311:57-67

43. Connally R, Veal D, Piper J (2004) Time-resolved fluorescence microscopy using an improved europium chelate BHHST for the in situ detection of Cryptosporidium and Giardia. Microsc Res Tech 64:312-322

44. Gudgin Dickson E, Pollak A, Diamandis E (1995) Time-resolved detection of lanthanide luminescence for ultrasensitive bioanalytical assays. J Photochem Photobiol B Biol 27:3-19

45. Mathis G (1993) Rare earth cryptates and homogeneous fluoroimmunoassays with human sera. Clin Chem 39(9):1953-1959

46. Mathis G (1995) Probing molecular interactions with homogeneous techniques based on rare earth cryptates and fluorescence energy transfer. Clin Chem 41(9):1391-1397

47. Enomoto K, Araki A, Nakajima T, Ohta H, Dohi K, Préaudat M, Seguin P, Mathis G, Suzuki R, Kominami G, Takemoto H (2002)
High-throughput miniaturized immunoassay for human interleukin-13 secreted from NK3.3 cells using homogenous time-resolved fluorescence. J Pharm Biomed Anal 28:73-79

48. Hemmilä I, Dakubu S, Mukkala V-M, Siitari H, Lövgren T (1984) Europium as a label in time-resolved immunofluorometric assays. Anal Biochem 127:335-343

49. Hemmilä I (1995) Luminescent lanthanide chelates - a way to more sensitive diagnostic methods. J Alloy Comp 225:480485

50. Hemmilä I, Mukkala V-M, Latva M, Kiilholma P (1993) Di- and tetracarboxylate derivatives of pyridines, bipyridines and terpyridines as luminogenic reagents for time-resolved fluorometric determination of terbium and dysprosium. J Biochem Biophys Methods 26:283-290

51. Smith D, Rossi C, Kijek T, Henchal E, Ludwig G (2001) Comparison of dissociation-enhanced lanthanide fluorescent immunoassays to enzyme-linked immunosorbent assays for detection of staphylococcal enterotoxin B, Yersinia pestis-Specific F1 antigen, and Venezuelan equine encephalitis virus. Clin Diagn Lab Immunol 8(6):1070-1075

52. Accardo S, Cutolo M (1986) Concentrations of lanthanides in plasma and synovial fluid in rheumatoid arthritis. Clin Chem 32 (8):1596

53. Diamandis E, Morton R, Reichstein E, Khosravi M (1989) Multiple fluorescence labeling with europium chelators. Application to time-resolved fluoroimmunoassays. Anal Chem 61(1):48-53

54. Scorilas A, Diamandis E (2000) Polyvinylamine-streptavidin complexes labeled with a europium chelator: a universal detection reagent for solid-phase time resolved fluorometric applications. Clin Biochem 33(5):345-350

55. Scorilas A, Bjartell A, Lilja H, Moller C, Diamandis E (2000) Streptavidin-polyvinylamine conjugates labeled with a europium chelate: applications in immunoassay, immunohistochemistry, and microarrays. Clin Chem 46(9):1450-1455

56. Härmä H, Soukka T, Lönnberg S, Paukkunen J, Tarkkinen $P$, Lövgren T (2000) Zeptomole detection sensitivity of prostatespecific antigen in a rapid microtitre plate assay using timeresolved fluorescence. Luminescence 15:351-355

57. Soukka T, Antonen K, Härmä H, Pelkkikangas A-M, Huhtinen P, Lövgren T (2003) Highly sensitive immunoassay of free prostatespecific antigen in serum using europium(III) nanoparticle label technology. Clin Chim Acta 328:45-58

58. Valanne A, Huopalahti S, Soukka T, Vainionpää R, Lövgren T, Härma H (2005) A sensitive adenovirus immunoassay as a model for using nanoparticle label technology in virus diagnostics. J Clin Virol 33:217-223

59. Valanne A, Huopalahti S, Vainionpää R, Lövgren T, Härmä H (2005) Rapid and sensitive HBsAg immunoassay based on fluorescent nanoparticles labels and time-resolved detection. J Virol Methods 129:83-90

60. Jaakohuhta S, Härmä H, Tuomola M, Lövgren T (2007) Sensitive Listeria spp. immunoassay based on europium(III) nanoparticle labels using time-resolved fluorescence. Int $\mathrm{J}$ Food Microbiol 114:288-294

61. Tang S, Moayeri M, Chen Z, Harma H, Zhao J, Hu H, Purcell R, Leppla S, Hewlett I (2009) Detection of anthrax toxin by an ultrasensitive immunoassay using europium nanoparticles. Clin Vaccine Immunol 16(3):408-413

62. Myyryläinen T, Talha S, Swaminathan S, Vainionpää R, Soukka T, Khanna N, Pettersson K (2010) Simultaneous detection of human immunodeficiency virus 1 and hepatitis $B$ virus infections using a dual-label time-resolved fluorometric assay. J Nanobiotechnol 8:27

63. Huhtinen P, Kivelä M, Kuronen O, Hagren V, Takalo H, Tenhu H, Lövgren T, Härmä H (2005) Synthesis, characterization, and 
application of $\mathrm{Eu}(\mathrm{III}), \mathrm{Tb}(\mathrm{III}), \mathrm{Sm}(\mathrm{III})$, and $\mathrm{Dy}(\mathrm{III})$ lanthanide chelate nanoparticle labels. Anal Chem 77:2643-2648

64. Wang F, Tan W, Zhang Y, Fan X, Wang M (2006) Luminescent nanomaterials for biological labelling. Nanotechnology 17:R1-R13

65. Murray K, Cao Y-C, Ali S, Hanley Q (2010) Lanthanide doped silica nanoparticles applied to multiplexed immunoassays. Analyst 135:2132-2138

66. Veiopoulou C, Lianidou E, Ioannou P, Efstathiou C (1996) Comparative study of fluorescent ternary terbium complexes. Application in enzyme amplified fluorometric immunoassay for afetoprotein. Anal Chim Acta 335:177-184

67. Lianidou E, Ioannou P, Sacharidou E (1994) Second derivative synchronous scanning fluorescence spectrometry as a sensitive detection technique in immunoassays. Application to the determination of a-fetoprotein. Anal Chim Acta 290:159-165

68. Christopoulos T, Diamandis E (1992) Enzymatically amplified time-resolved fluorescence immunoassay with terbium chelates. Anal Chem 64(4):342-346

69. Bathrellos L, Lianidou E, Ioannou P (1998) A highly sensitive enzyme-amplified lanthanide luminescence immunoassay for interleukin 6. Clin Chem 44(6):1351-1353

70. Yu H, Diamandis E (1993) Ultrasensitive time-resolved immunofluorometric assay of prostate-specific antigen in serum and preliminary clinical studies. Clin Chem 39(10):2108-2114

71. Zheng X-Y, Lu J-Z, Zhu Q-Z, Xu J-G, Li Q-G (1997) Study of a lanthanide fluorescence system with a coupled reaction based on hemin catalysis. Analyst 122:455-458

72. Qin Q-P, Peltola O, Pettersson K (2003) Time-resolved fluorescence resonance energy transfer assay for point-of-care testing of urinary albumin. Clin Chem 49(7):1105-1113

73. Kokko L, Sandberg K, Lövgren T, Soukka T (2004) Europium(III) chelate-dyed nanoparticles as donors in a homogeneous proximitybased immunoassay for estradiol. Anal Chim Acta 503:155-162

74. Kokko L, Kokko T, Lövgren T, Soukka T (2008) Particulate and soluble $\mathrm{Eu}(\mathrm{III})$-chelates as donor labels in homogeneous fluorescence resonance energy transfer based immunoassay. Anal Chim Acta 606:72-79

75. Valanne A, Lindroos H, Lövgren T, Soukka T (2005) A novel homogeneous assay format utilising proximity dependent fluorescence energy transfer between adjacent particulate labels. Anal Chim Acta 539:251-256

76. Scaff W Jr, Dyer D, Mori K (1969) Fluorescent europium chelate stain. J Bacteriol 98(1):246-248

77. Kemper C, Berggren K, Diwu Z, Patton W (2001) An improved, luminescent europium-based stain for detection of electroblotted proteins on nitrocellulose or polyvinylidene difluoride membranes. Electrophoresis 22:881-889

78. Beverloo H, van Schadewijk A, van Gelderenboele S, Tanke H (1990) Inorganic phosphors as new luminescent labels for immunocytochemistry and time-resolved microscopy. Cytometry 11:784-792

79. Seveus L, Väisälä M, Syrjänen S, Sandberg M, Kuusisto A, Harju R, Salo J, Hemmilä I, Kojola H, Soini E (1992) Time- resolved fluorescence imaging of europium chelate label in immunohistochemistry and in situ hybridization. Cytometry 13:329-338

80. Sevéus L, Väisälä M, Hemmilä I, Kojola H, Roomans G, Soini E (1994) Use of fluorescent europium chelates as labels in microscopy allows glutaraldehyde fixation and permanent mounting and leads to reduced autofluorescence and good long-term stability. Microsc Res Tech 28:149-154

81. Rulli M, Kuusisto A, Salo J, Kojola H, Simell O (1997) Timeresolved fluorescence imaging in islet cell autoantibody quantitation. J Immunol Methods 208:169-179

82. Väisänen V, Härmä H, Lilja H, Bjartell A (2000) Time-resolved fluorescence imaging for quantitative histochemistry using lanthanide chelates in nanoparticles and conjugated to monoclonal antibodies. Luminescence 15:389-397

83. Connally R, Veal D, Piper J (2002) High resolution detection of fluorescently labeled microorganisms in environmental samples using time-resolved fluorescence microscopy. FEMS Microbiol Ecol 41:239-245

84. Connally R, Jin D, Piper J (2006) High intensity solid-state uv source for time-gated luminescence microscopy. Cytometry 69A: $1020-1027$

85. Deng W, Jin D, Drozdowicz-Tomsia K, Yuan J, Goldys E (2010) Europium chelate (BHHCT- $\mathrm{Eu}^{3+}$ ) and its metal nanostructure enhanced luminescence applied to bioassays and time-gated bioimaging. Langmuir 26(12):10036-10043

86. Marriott G, Heidecker M, Diamandis E, Yan-Marriott Y (1994) Time-resolved delayed luminescence image microscopy using an europium ion chelate complex. Biophys J 67:957-965

87. de Haas R, Verwoerd N, van der Corput M, van Gijlswijk R, Siitari H, Tanke H (1996) The use of peroxidase-mediated deposition of biotin-tyramide in combination with time-resolved fluorescence imaging of europium chelate label in immunohistochemistry and in situ hybridization. J Histochem Cytochem 44 (10):1091-1099

88. Jürgens G, Hermann A, Aktuna D, Petek W (1992) Dissociationenhanced lanthanide fluorescence immunoassay of lipoprotein(a) in serum. Clin Chem 38(6):853-859

89. Bacigalupo M, Meroni G, Secundo F, Scalera C, Quici S (2009) Antibodies conjugated with new highly luminescent $\mathrm{Eu}^{3+}$ and $\mathrm{Tb}^{3+}$ chelates as markers for time resolved immunoassays. Application to simultaneous determination of clenbuterol and free cortisol in horse urine. Talanta 80:954-958

90. Wortberg M, Cammann K (1993) Determination of triazine herbicides with time-resolved fluoroimmunoassay using europium(III)-chelates. Fresenius J Anal Chem 346:757-760

91. Diamandis E, Papanastasiou-Diamandi A, Lustig V, Khosravi M, Tan A (1989) Time-resolved immunofluorometric assay of human pancreatic isoamylase in serum, with use of two monoclonal Antibodies. Clin Chem 35(9):1915-1920

92. Papanastasiou-Diamandi A, Conway K, Diamandis E (1989) Digoxin immunoassay with monoclonal and polyclonal antibodies using time-resolved fluorometry. J Pharm Sci 78(8):617-621 